\author{
Peter Friedrich, Diana Eerma ${ }^{1}$
}

\title{
Ein Kontenrahmen für eine ,gesellschaftsbezogene Rechnungslegung“' der Fakultät für Volks- und Betriebswirtschaftslehre der Universität Tartu in Estland
}

\section{Problemstellung}

Zurzeit werden in Deutschland die Rechnungslegung und Buchführung von Universitäten auf das kaufmännische Rechnungswesen umgestellt (Bauder/Jungen 2009). ${ }^{2}$ Den Universitäten in Deutschland und Estland fehlen ein für die Feststellung des gesellschaftlichen Erfolges von Forschung und Lehre geeignetes Rechnungswesen und ein Buchführungssystem zur gesellschaftsbezogenen Erfolgsermittlung. Mittels des gesellschaftsbezogenen Rechnungswesens soll der soziale Erfolg, den eine Wirtschaftseinheit bewirkt, z. B. eine Universität, festgestellt werden. Deshalb sind in dieser Studie folgende Probleme zu diskutieren:

1. Welche Versuche gesellschaftsbezogener Rechnungslegung existieren? Welche Bewertungsbasis kann für ein gesellschaftsbezogenes Rechnungswesen und eine adäquate Buchführung gewählt werden?

2. Welcher Kontenrahmen resultiert und wie ist zu buchen?

3. Welche Resultate ergeben sich für die Fakultät für Volks- und Betriebswirtschaftslehre der Universität Tartu für das Jahr 2006?

4. Welche Grenzen der Aussagefähigkeit sowie Notwendigkeiten der Fortentwicklung sind zu berücksichtigen?

Im Mittelpunkt der Ausführungen steht die Entwicklung eines Buchführungssystems für ein solches gesellschaftliches Rechnungswesen. Zwar existieren etliche Beispiele und Zusammenstellungen sozialer Vor- und Nachteile (z. B. Fischer-Winkelmann 1980; Kaya/Yayla 2007; Gray/ Bebbington/Gray 2010) sowie Berichte über gesellschaftliche Erfolge von Firmen, aber ein gesellschaftsbezogenes und integriertes Rechnungswesen für eine Universität oder eine Fakultät

1 Prof. Dr. Dr. h.c. Peter Friedrich (em.), Senior Researcher, Universität Tartu, Fakultät für Volks- und Betriebswirtschaftslehre, Narva 4, 51009 Tartu/Estland; Tel.: 00372/7376350, Peter.Friedrich@mtk.ut.ee.

Diana Eerma, Researcher, Universität Tartu, Fakultät für Volks- und Betriebswirtschaftslehre, Narva 4, 51009 Tartu/Estland; Tel.: 00372/7376344, Diana.Eerma@mtk.ut.ee. Die Studie wurde gefördert mit Mitteln des estnischen Ministeriums für Wissenschaft und Erziehung im Rahmen des Projektes „Pfadabhängige Innovationen in einem kleinen Land" sowie mit Mitteln des Europäischen Strukturfonds.

2 Verschiedentlich verspricht man sich verbesserte Informationen über die Vermögenslage einer Hochschule, eine Abkehr von einer dominanten inputorientierten Steuerung, erhöhte Transparenz, verursachungsgerechte Betrachtung von Vermögensänderungen und eine erhöhte Vergleichbarkeit zwischen öffentlichen Institutionen (Küpper 2001; Bräuning 2004; Arbeitskreis Hochschulrechnungswesen der deutschen Universitätskanzler 1999; Waltenberger 2006) sowie den Ausweis von Gewinnen und Verlusten. 
fehlt bislang. In diesem Beitrag wird eine fakultätsspezifische Konzeption entwickelt und deren tatsächliche Anwendung für das Jahr 2006 auf der Grundlage erhobener Daten vorgeführt.

\section{Ansätze für ein gesellschaftsbezogenes Rechnungswesen}

Die Diskussion um eine gesellschaftsbezogene Rechnungslegung behandelt hauptsächlich Grundsatzfragen, wie und inwieweit sich gesellschaftlicher Erfolg überhaupt feststellen lässt (Eichhorn 1974; Fischer-Winckelmann 1980; Kaya/ Yayla ${ }^{4}$ 2007; Gray/Bebbington/Gray), in welcher Weise soziale Vor- und Nachteile von Maßnahmen in einzelnen Aufgabenbereichen, z. B. Umweltökonomie, Gesundheitsökonomik, Bildungsökonomik, ausgedrückt werden könnten und welche Wirtschaftssubjekte gesellschaftsbezogenes Rechnungswesen benutzen sollten. Vernachlässigt wurden einzelne Bildungsinstitutionen, z. B. eine Fakultät, und die Ermittlung des sozialen Erfolges aller Aktivitäten einer Fakultät während einer Periode.

In Tabelle 2 werden die Hauptansätze gesellschaftsbezogenen Rechnungswesens aufgeführt und einige grundlegende Quellen zitiert. Ferner werden dort in den Spalten für die Entwicklung des fakultätsorientierten und gesellschaftsbezogenen Rechnungswesen wesentliche Eigenheiten der bisherigen Ansätze angegeben. Sie informieren über die grundlegenden angewendeten Bewertungsverfahren für soziale Erfolge (Nutzen Kosten Analyse, Kostenwirksamkeitsanalyse oder Nutzwertanalyse). Sie lassen erkennen, ob gesellschaftliche Effekte Berücksichtigung finden, ob sie maßnahmen- und projektorientiert ausgerichtet sind oder ob sie die Gesamtaktivitäten einer Wirtschaftseinheit einbeziehen. Die Ausrichtung auf Privatunternehmen, öffentliche Unternehmen oder öffentliche Verwaltungen deuten ebenfalls auf die Eignung der Ansätze für das soziale Fakultätsrechnungswesen hin. Wichtig ist, inwieweit sie soziale Bewertungshilfen für Fakultätstransaktionen bieten und den in Tabelle 1 erwähnten grundlegenden Anforderungen für ein gesellschaftsbezogenes Buchführungssystem entsprechen. Schließlich wird auch angedeutet, ob sich die Ansätze für ein soziales Buchführungssystem der Fakultät eignen.

- Feststellung des gesellschaftlichen Erfolges für ein Jahr,

- eine möglichst vollständige Erfassung aller Transaktionen,

- Techniken zur Bewertung und Erfassung der Transaktionen,

- Konventionen zur einheitlichen Behandlung der Transaktionen für alle einbezogenen Institutionen,

- Zuordnung der gesellschaftlichen Erfolge und Misserfolge zu an den Transaktionen beteiligten Institutionen (Wirtschaftseinheiten),

- eine periodengerechte Spezifizierung der Transaktionen,

- eine gleichförmige Dimensionierung der Transaktionen,

- ein gemeinsames und spezifiziertes Zuordnungs- und Gruppierungssystem,

- ein periodifiziertes, finanzielles Buchhaltungssystem zur Erfassung von Erfolgs- und Bestandsänderungen,

- geeignete Verknüpfungen mit anderen Buchhaltungssystemen.

Tabelle 1: Anforderung an eine gesellschaftsbezogene jährliche Rechnungslegung

Quelle: Zusammenstellung der Autoren, Friedrich 1991

3 Mit vielen europäischen Quellenangaben.

4 Mit einem ,survey“ englischer Literatur. 
Alle Ansätze zeigen relevante soziale Effekte auf. Insbesondere die ökologischen, gesundheitlichen und erzieherischen Ansätze bieten Hinweise zur Bewertung der gesellschaftlichen Wirkungen der Fakultät. Allerdings sind einige Ansätze vornehmlich projekt- und maßnahmenorientiert. Jene, die die Gesamtaktivitäten von Wirtschaftseinheiten zum Gegenstand haben, betreffen meist private oder öffentliche Unternehmen und weniger öffentliche Verwaltungen. Vollständige Buchführungssysteme sind selten und kommen meist den Anforderungen der Tabelle 1 nicht nach. Besonders die kostenwirksamkeits- und nutzwertbasierten Ansätze eignen sich wenig, um die Beiträge einzelner Wirtschaftseinheiten zu isolieren und zum gesellschaftlichen Gesamterfolg zu bestimmen. Ansätze mit existierender Buchführung werden zur gesellschaftlichen Fakultätsrechnung ausgebaut. 
Peter Friedrich und Diana Eerma

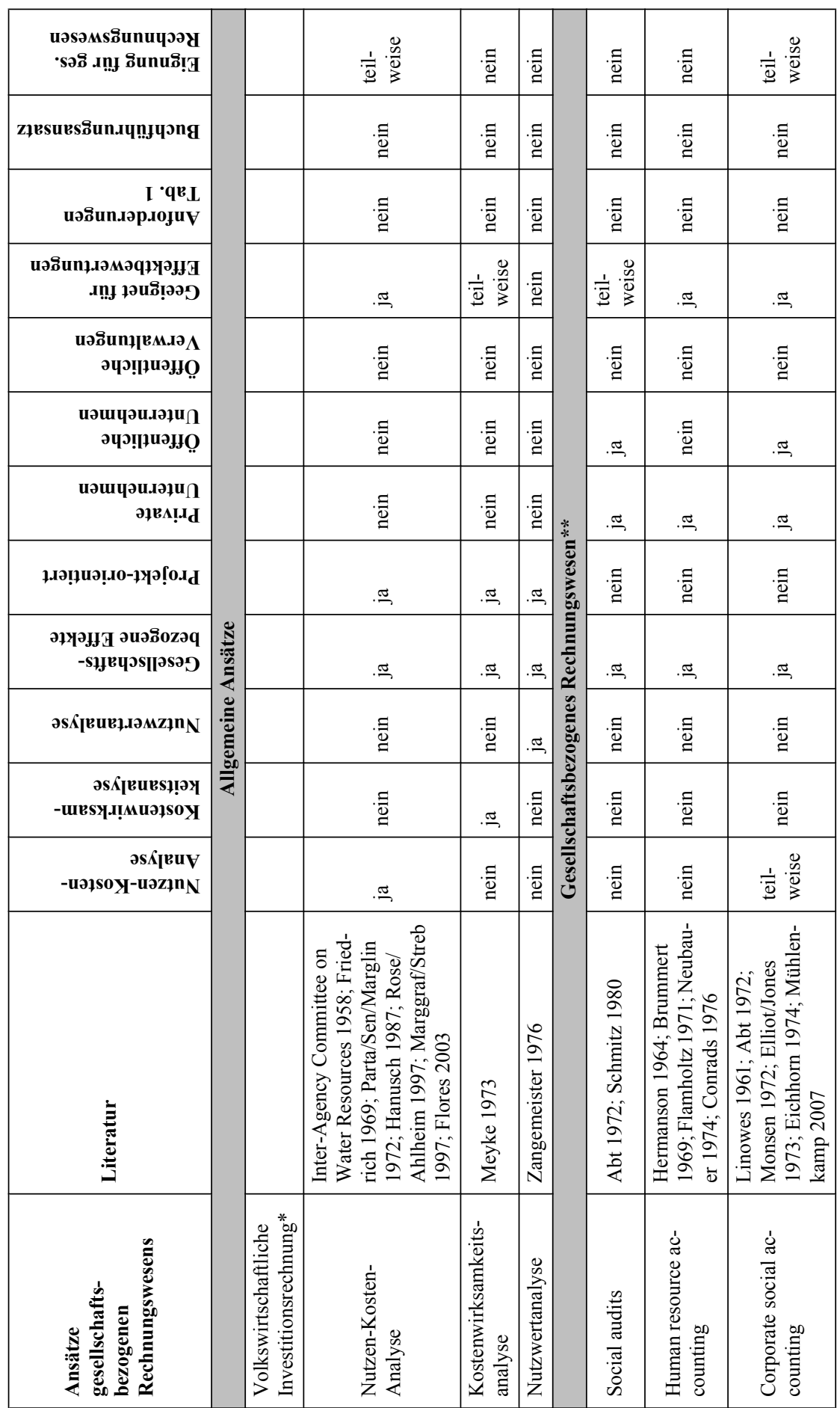


Ein Kontenrahmen für eine "gesellschaftsbezogene Rechnungslegung"

\begin{tabular}{|c|c|c|c|c|c|c|c|c|c|}
\hline : & : & & $\frac{.}{\vec{D}}$ & 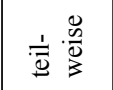 & $\frac{1}{\frac{m}{D}}$ & 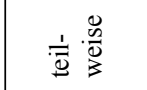 & $\frac{1}{\Phi} \cdot \frac{\infty}{d}$ & 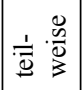 & 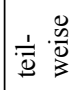 \\
\hline$\stackrel{\Xi}{\Xi}$ & $\stackrel{\Xi}{\stackrel{\Xi}{巳}}$ & & 寻 & 寻 & 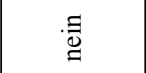 & $\stackrel{\Xi}{\Xi}$ & $\stackrel{\Xi}{\stackrel{\Xi}{\rightleftarrows}}$ & $\stackrel{\Xi}{\Xi}$ & $\stackrel{\Xi}{\Xi}$ \\
\hline$\stackrel{\Xi}{\Xi}$ & $\stackrel{\Xi}{\Xi}$ & & 氖 & : & : & $\stackrel{\Xi}{\Xi}$ & $\stackrel{\Xi}{\Xi}$ & $\stackrel{\Xi}{\Xi}$ & 寻 \\
\hline.$\approx$ & 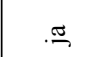 & & . & 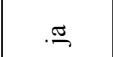 & . &.$\approx$ & . &.$\frac{\pi}{2}$ & . \\
\hline$\stackrel{\Xi}{\rightleftarrows}$ & $\frac{0}{\frac{m}{0}}$ & & $\stackrel{\Xi}{\simeq}$ & $\stackrel{\pi}{\sim}$ & $\cong$ & $\stackrel{\pi}{\sim}$ & $\stackrel{\pi}{\sim}$ & . & $\stackrel{\Xi}{\Xi}$ \\
\hline$\stackrel{\pi}{.}$ & . & & . & . &.$\pi$ &.$\underset{\pi}{\sim}$ & . & . & : \\
\hline.$\stackrel{\varpi}{\Omega}$ &.$\stackrel{\varpi}{\Omega}$ & 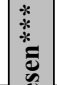 & 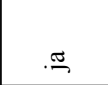 & 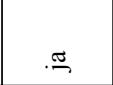 &.$\approx$ & 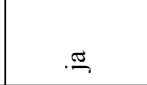 & . &.$\stackrel{\sigma}{.}$ & $\stackrel{\Xi}{\stackrel{\Xi}{\Xi}}$ \\
\hline$\stackrel{\Xi}{\Xi}$ & 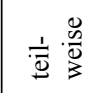 & 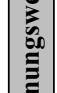 & . & 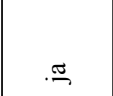 & $\frac{1}{\Phi} \frac{\infty}{d}$ & $\frac{1}{\Phi} \cdot \frac{\infty}{\Phi}$ & $\frac{1}{\Phi} \cdot \frac{\infty}{0}$ &.$\ddot{g}$ &.$\approx$ \\
\hline.$\cong$ &.$\cong$ & 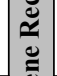 & 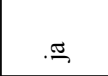 & 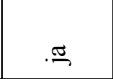 & 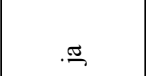 & 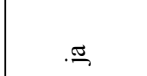 & . & 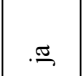 & . \\
\hline 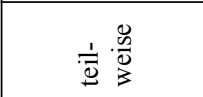 & $\stackrel{\Xi}{\Xi}$ & $\begin{array}{c}0 \\
0 \\
0 \\
0 \\
0 \\
0 \\
0\end{array}$ & 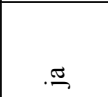 & 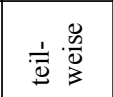 & $\stackrel{\Xi}{\Xi}$ & $\stackrel{\Xi}{\Xi}$ &.$\approx$ & 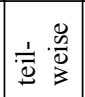 & $\frac{1}{\bar{\Phi}} \cdot \frac{\sqrt[m]{0}}{3}$ \\
\hline 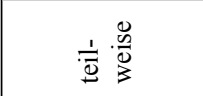 & $\stackrel{\Xi}{\Xi}$ & 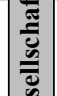 &.$\underset{0}{.}$ &.$\stackrel{.}{.}$ & $\stackrel{\Xi}{\stackrel{\Xi}{\rightleftarrows}}$ &.$\underset{\pi}{\sim}$ & $\stackrel{\Xi}{\Xi}$ & $\frac{0}{\frac{m}{2}}$ & 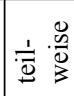 \\
\hline$\stackrel{\Xi}{\circlearrowright}$ & $\stackrel{\Xi}{\underset{\Xi}{\Xi}}$ & 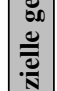 & 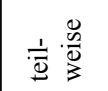 & $\frac{1}{\bar{D}} \cdot \frac{\tilde{n}}{\tilde{D}}$ &.$\underset{\sim}{.}$ & 寻 & : & 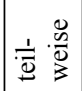 & $\stackrel{\Xi}{\Xi}$ \\
\hline 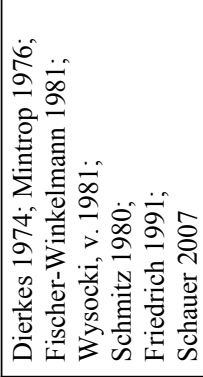 & 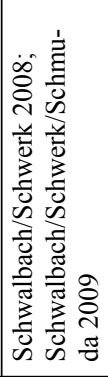 & के & 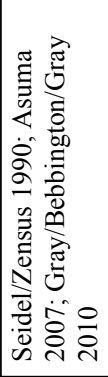 & 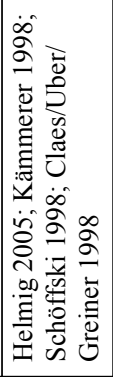 & 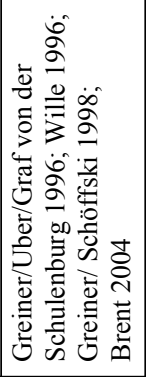 & 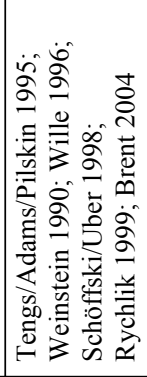 & 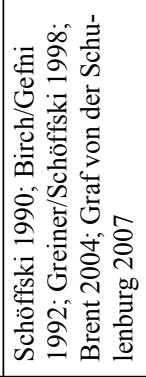 & 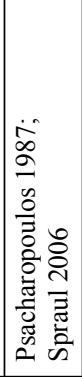 & 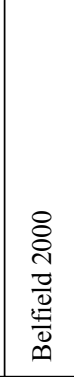 \\
\hline 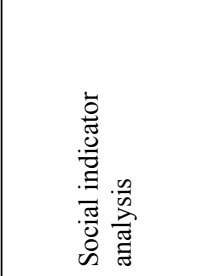 & 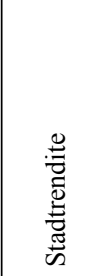 & & 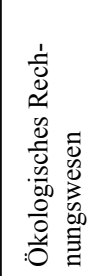 & 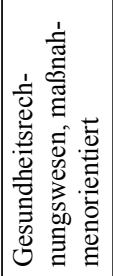 & 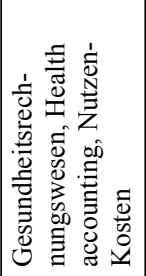 & 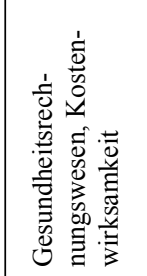 & 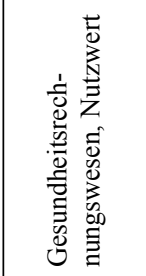 & 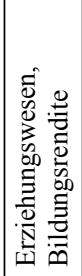 & 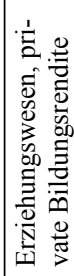 \\
\hline
\end{tabular}


Peter Friedrich und Diana Eerma

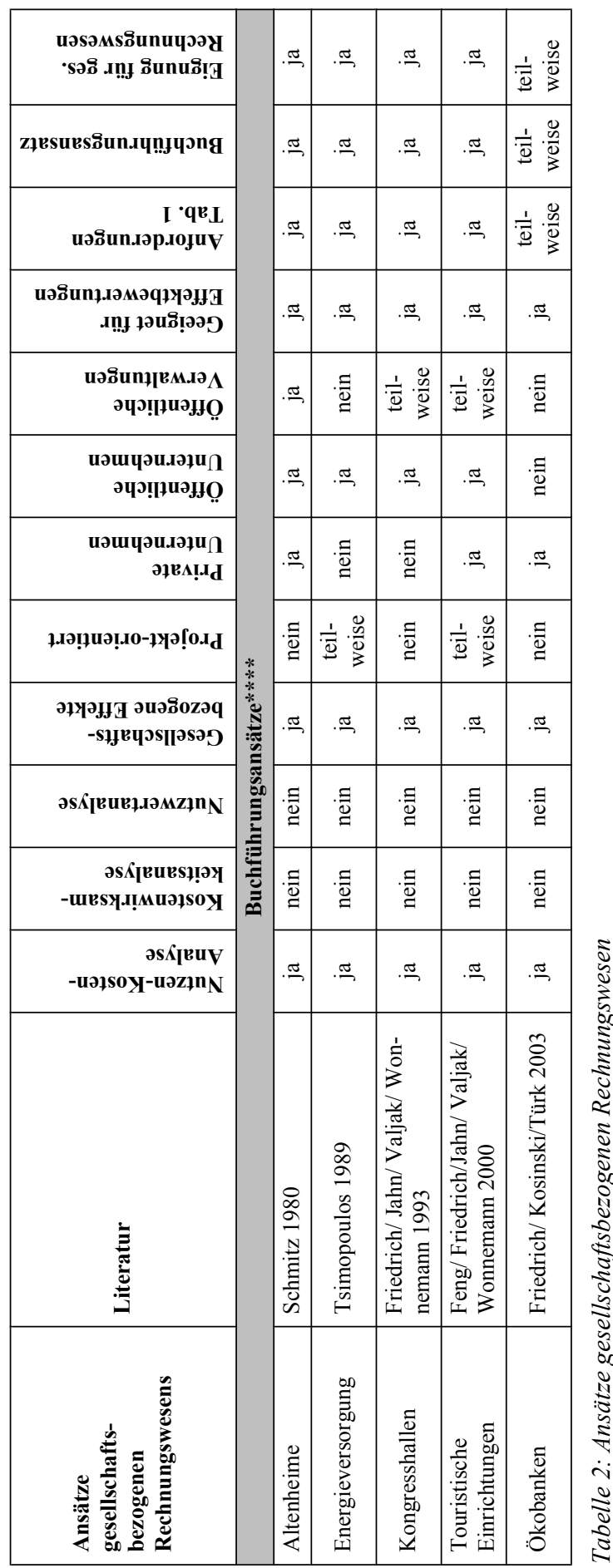

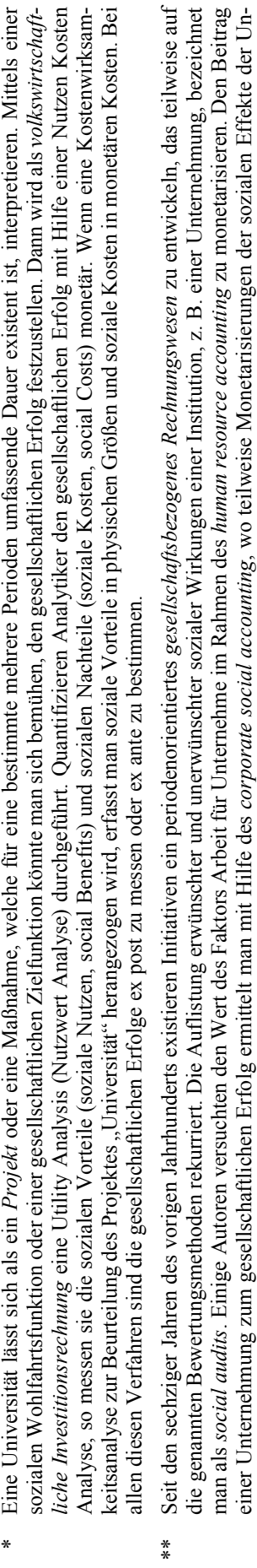


Ein Kontenrahmen für eine "gesellschaftsbezogene Rechnungslegung"
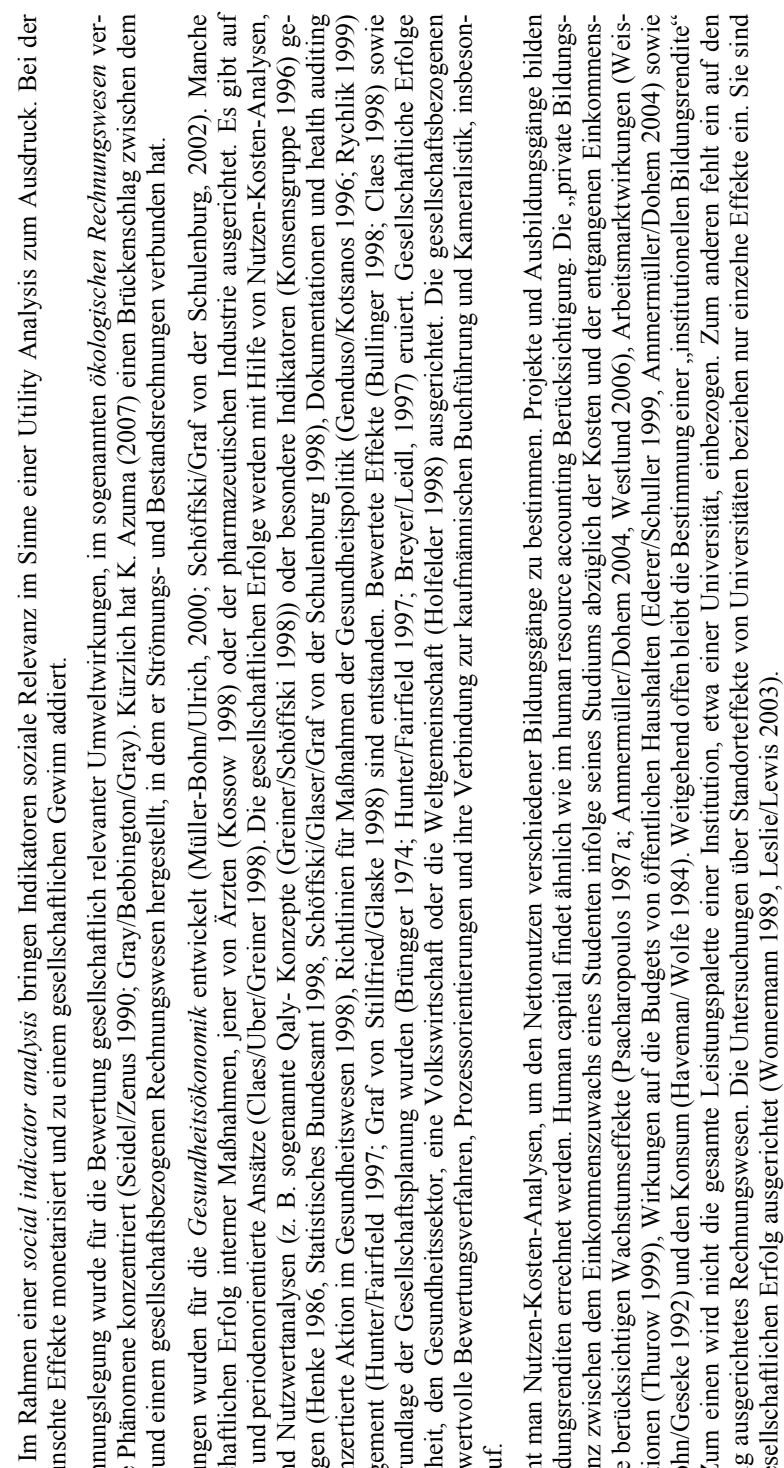

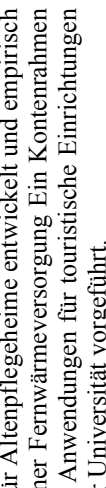

运

站娄

战证

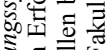

를

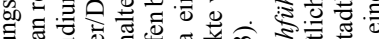

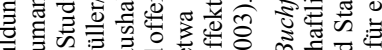

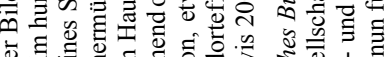

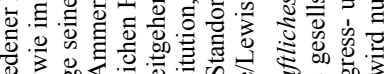

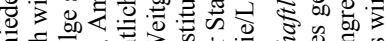

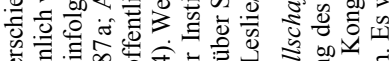

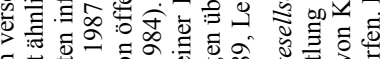

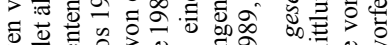

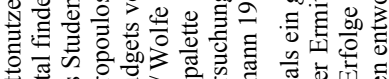

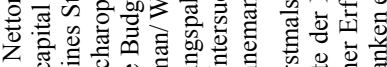

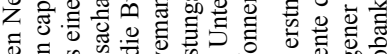

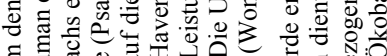

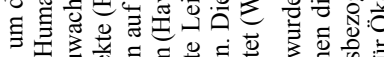

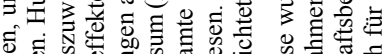

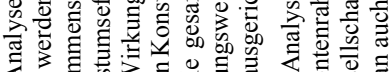

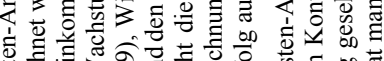

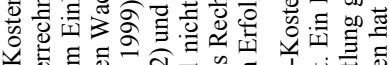

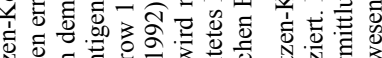

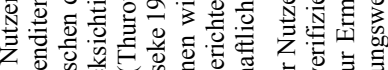

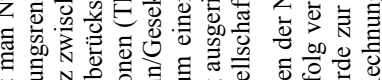

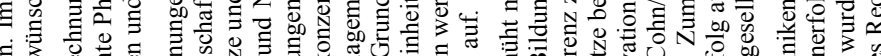

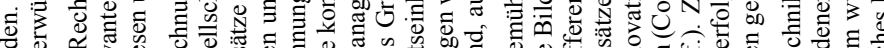

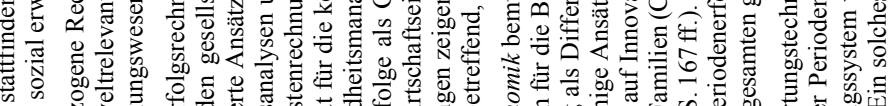

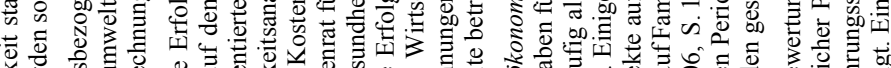

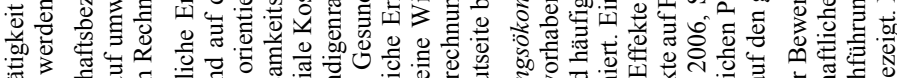

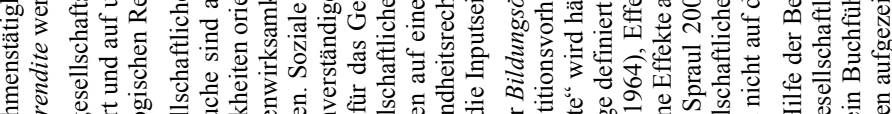

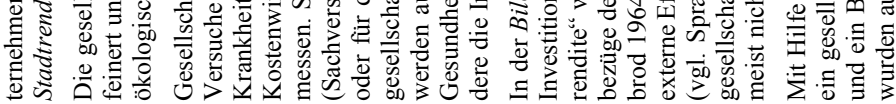




\section{Grundeigenheiten der gesellschaftsbezogenen Rechnungslegung der Fakultät}

\section{Basisansatz}

Der in den Grundzügen für andere Wirtschaftssubjekte ermittelte Kontenrahmen des gesellschaftsbezogenen Rechnungswesens wird auf eine Fakultät übertragen. Dafür sind einige Grundentscheidungen zu treffen (Friedrich 1991, S. 197 ff.).

Eine soziale Wohlfahrtsfunktion existiert für die Fakultät für Volks- und Betriebswirtschaftslehre nicht. Deshalb muss das gesellschaftsbezogene Rechnungswesen an einer Nutzen-Kosten-Analyse, einer Kostenwirksamkeitsanalyse oder einer Nutzwertanalyse anknüpfen. Bei letzterer bringen soziale Indikatoren die Nutzwerte zum Ausdruck. Die gesellschaftlichen Vor- und Nachteile können in unterschiedlichen Dimensionen erfasst werden, z. B. monetären, zeitlichen oder physischen Größen (Studentenzahlen, Veranstaltungsmengen). Da viele Informationen im kommerziellen Rechnungswesen der Fakultät in Geldeinheiten zur Verfügung stehen, entwickeln wir ein Buchführungs- und Kontensystem für eine Rechnung in Geldgrößen. Auf der Grundlage des Zahlungsbereitschaftsansatzes ${ }^{5}$ fixieren wir die monetarisierten sozialen Vor- (soziale Nutzen, Benefits) und Nachteile (soziale Kosten, Costs).

Direkte, marktorientierte soziale Nutzen werden mittels der Konsumentenrenten und der Erlöse ausgedrückt. Indirekt werden positive externe Effekte bewertet, z. B. als Einkommenserhöhungen, Geldwerte von Zeitersparnissen, Kostenersparnisse, geringere Versicherungsentschädigungen, Ersparnisse anderer Institutionen, verminderte Versicherungsbeiträge, höhere Werte von unterstellten Erlösen und Konsumentenrenten, die mit Hilfe hypothetischer Nachfragefunktionen ermittelt werden (Inter-Agency Committee on Water Resources 1958; Friedrich 1969; Parta/Sen/ Marglin 1972; Hanusch 1987; Ahlheim/Rose 1989; Flores 2003 und die dortigen Quellenangaben). Weitere indirekte Bewertungen externer Effekte drücken Erhöhungen von Vermögenswerten, Änderungen von Leasingbeträgen sowie Geldvorteile aus externen Effekten aufgrund verbesserter Fakultätsdienstleistungen aus.

Soziale Kosten werden direkt über Kosten und Einkaufsvorteile (input orientierte Produzentenrente) bewertet. Der Bewertung negativer externer Effekte dienen die indirekten Bewertungsmethoden.

Perioden überdauernde soziale Vorteile und Nachteile werden als Bestände an sozialen Nutzen (social assets, Vorteilsbestände, soziale Vermögen) und sozialen Kosten (social liabilities, Nachteilsbestände, soziale Verbindlichkeiten) definiert. Bei deren Bewertung ist zu beachten, welche Generation Vor- und Nachteile erfährt. Im Kontenrahmen berücksichtigen wir deshalb betreffende Zeithorizonte, für die Betroffenen. Obwohl die Zahlungsbereitschaft zukünftiger Generationen zu berücksichtigen wäre, verbleibt nur die Möglichkeit, die gegenwärtige Generation für zukünftige Generationen werten zu lassen.

5 Beim Zahlungsbereitschaftsansatz werden die gesellschaftlichen Vorteile (soziale Nutzen) mit dem maximalen Betrag gemessen, den die Empfänger für die Bereitstellung einer Leistung zu zahlen bereit sind. Die Nachteile (soziale Kosten) werden mit dem Geldbetrag ausgedrückt, den die Benachteiligten bereit sind, zur Verhinderung der Bereitstellung einzusetzen (vgl. obige Literaturangaben). 
Schwierig ist die Bestimmung der Personengruppe, deren Zahlungsbereitschaft ermittelt werden soll. (Friedrich, Jutila 2001). Da die Fakultätsaktivitäten sich auf Estland konzentrieren, sollte die Wohlfahrt der Einwohner Estlands erhöht werden und nicht eine übernationale Wohlfahrtsänderung betrachtet werden. Jedoch beruht die Bewertung gemäß Zahlungsbereitschaftsansatz auf Preisen und Marktwerten. Einige Fakultätsleistungen, z. B. Forschungsleistungen, werden europaweit bestimmt oder beziehen Universitäten oder Leistungsempfänger sowie Marktprozesse in anderen Staaten ein. Auch die europäischen Kapitalmärkte sind integriert. Deshalb berücksichtigen wir Personen, die von den Leistungen real oder monetär betroffen werden, bei der Anwendung des Zahlungsbereitschaftsansatzes.

Dies ist unbefriedigend, da einige Nationen oder regionale Entscheidungsträger nur an der Wohlfahrt ihrer Staatsbürger oder Einwohner interessiert sind und wenig Interesse an Vor- und Nachteilen in anderen Ländern zeigen. Ein solches Verhalten ist bei regionaler Konkurrenz nahe liegend (Friedrich/Jutila 2001). Entsprechende Bewertungsmethoden für Nutzen-Kosten-Analysen existieren kaum. Die Region, in der Wohlfahrtsänderungen auftreten, ist meist jene, in der eine Fakultät mit ihren Beschaffungs-, Leistungserstellungs-, Abgabe- und Finanzierungsaktivitäten eingebettet ist und wo sich Fakultätsaktivitäten bemerkbar machen, z. B. Narva, Pärnu, Tartu, Tallin und andere estnische Orte. Deshalb wählen wir Estland als Analyseregion.

Infolge enormer Informationsprobleme werden Folgetransaktionen und Effekte nur für solche Aktivitäten betrachtet, die direkt mit der Fakultät verbunden sind. Damit werden auch die Zahl der einbezogenen Personen sowie Wirtschaftseinheiten und die Regionsgröße beschränkt.

Soziale Nutzen sowie soziale Kosten lassen sich gemäß den sozialen Wirkungen der Fakultätsaktivitäten gruppieren. Soziale Vermögen und soziale Verpflichtungen werden gemäß ihren langfristigen Effekten zusammengefasst. Unsere Gruppierung ist vornehmlich an den Fakultätsaktivitäten, z. B. Lehre, Forschung, Konferenzgestaltung, wissenschaftliche Kooperation, der open University, berufsorientierter Ausbildung, Fortbildung sowie Beratung orientiert. Obwohl unser Kontenrahmen fakultätsspezifisch entwickelt wurde, lässt er sich in jene anderer Institutionen integrieren.

Um Doppelerfassungen zu vermeiden, rechnen wir soziale Nutzen und soziale Kosten den Wirtschaftseinheiten zu, die infolge ihres Zusammenwirkens die sozialen Effekte bewirken. Zuordnungskriterien waren zu entwickeln, um jene soziale Nutzen und soziale Kosten, welche nicht von der Fakultät bewirkt werden, auszugrenzen. Für andere an sozialen Nutzen und sozialen Kosten mitwirkenden Wirtschaftseinheiten, sind Abgrenzungskonten vorgesehen. Der Kontenrahmen betrifft die Fakultät und die verbunden Colleges. Zur Vereinfachung wurde angenommen, dass jene soziale Nutzen und soziale Kosten, die sich im kaufmännischen Rechnungswesen der Fakultät niederschlagen, von der Fakultät ausgegangen sind.

Die sogenannte Alternativsituation bereitet bei der Wohlfahrtsmessung beachtliche Probleme (Friedrich 1991, S. 202 ff.). Im kaufmännischen Rechnungswesen wird als Alternativsituation die Nichtexistenz einer Firma oder ein Gewinn von Null angenommen. Im Falle einer Fakultät könnte jedoch eine andere Institution die Aufgabenbewältigung übernehmen. Die ermittelten sozialen Nutzen und Kosten könnten ebenfalls auftreten. Der eigentliche Beitrag der Fakultät würde in der Netto-Nutzen-Differenz zur nächsten weniger erfolgreichen Organisationslösung bestehen. Als Approximation wird unterstellt, dass der soziale Nettonutzen der Fakultät nicht entsteht, falls die Fakultät nicht vorhanden ist. 
Die Ausgestaltung des Kontenrahmens erfolgt unter Rückgriff auf das Abschlussprinzip ${ }^{6}$ das insbesondere die Beziehungen zu anderen Wirtschaftseinheiten verdeutlicht, und der Doppik, die auch in der kaufmännischen Buchführung Verwendung findet, um Bestands- und Strömungsgrößen zu buchen.

In Übereinstimmung mit unseren Grundannahmen über die Ausgestaltung der gesellschaftsbezogenen Rechnungslegung und ihres Kontenrahmens wird die folgende Grundbeziehung formuliert:

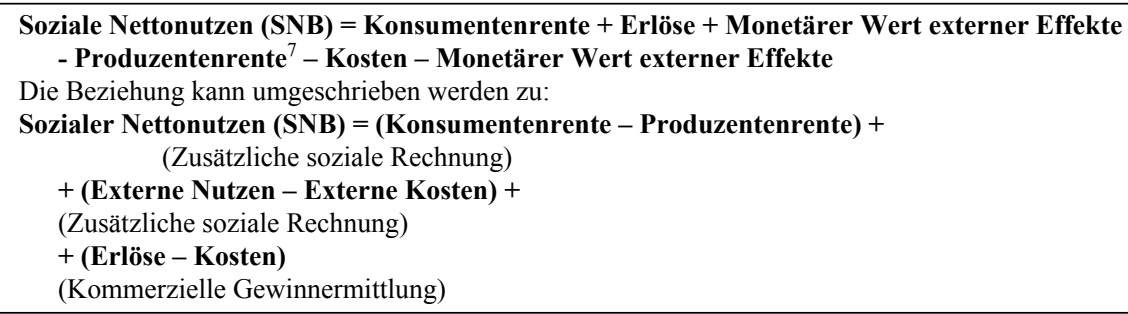

Auf dieser Grundlage lässt sich eine gesellschaftsbezogene Rechnungslegung entwickeln. Sie umfasst:

1. Gesellschaftliche Rechnung = zusätzliche soziale Rechnung + kommerzielle Rechnung

2. Laufender Nettonutzen: = zusätzliche soziale Nutzen und Kosten Rechnung (Konto) + kommerzielle Gewinn und Verlust Rechnung (Konto)

3. Gesamtgesellschaftliche Bilanz $=$ zusätzliche soziale Bilanz + kommerzielle Bilanz

Da die kaufmännische Buchführung den Managern in privat und öffentlich rechtlichen Institutionen recht bekannt ist, konzentrieren wir uns auf die zusätzliche Rechnungslegung (in (1.) und auf die gesamtgesellschaftliche Bilanz (3.), in der die zusätzliche soziale Bilanz und die kommerzielle Bilanz zusammengeführt werden.

\section{Aufgaben und Aktivitäten der Fakultät für Volks- und Betriebswirtschaftslehre der Universität Tartu}

Die Ausgestaltung des Kontenrahmens wird besonders von den Aufgaben und Aktivitäten der Fakultät geprägt (vgl. Faculty of Economics and Business Administration 2007). Die öffentlich rechtliche Universität Tartu soll forschungsgestützt Wissenschaftler ausbilden, die Lehrfortschritte in allen ihren Aktivitätsfeldern ermöglichen und Ausbildungs-, Forschungs- und Entwicklungsleistungen der Gesellschaft zur Verfügung stellen.

Die Aktivitäten der Fakultät für Volks- und Betriebswirtschaftslehre werden hauptsächlich aus Semestergebühren, Zuweisungen seitens der Zentralregierung, Zuweisungen aus dem Universi-

6 Einige Ausgestaltungsprinzipien von Kontenrahmen sollen den Ablauf des Produktionsprozesses aufzeigen, z. B. um zu verdeutlichen, wie und an welchen Stellen soziale Kosten entstehen.

7 Die Produzentenrente bezeichnet hier ein Produzentenvorteil, der dadurch entsteht, dass der Produzent billiger einkaufen kann als auf Faktormärkten mit vollständiger Konkurrenz. 
tätshaushalt und aus eingeworbenen Forschungsgeldern aus Projekten der Ministerien, der Europäischen Union, von Firmen, von Forschungsfonds sowie aus Beratungstätigkeit finanziert. Die Fakultät agiert wie ein öffentliches Unternehmen. Sie setzt ihr finanzielles, absatz-, produktionsund beschaffungspolitisches Instrumentarium ein und arbeitet mit Organisationseinheiten der Universität zusammen. Sie betrachtet die eng verbundenen Colleges in Pärnu, in Narva und das Europakolleg als Leistungsabnehmer oder Klienten. Die Fakultät besitzt viele Kompetenzen und Entscheidungsmöglichkeiten. Dies trifft auch für die Colleges zu.

Die Hauptaufgaben haben die staatlich oder nicht-staatlich finanzierte Gewinnung und die Vermittlung von Wissen zum Gegenstand. In der Lehre werden Bachelor- und Masterabschlüsse seitens der Studenten erworben. Berufsorientiertes Wissen vermitteln die Colleges. Fernstudien existieren an der Fakultät und den Colleges ebenfalls. Die Fakultät unterstützt die Forschungen der Doktoranden und bietet ein Doktorandenstudium an. Eine ihrer Hauptaufgaben bildet die Forschungstätigkeit und die Unterstützung des Werdeganges von Wissenschaftlern und zukünftigen Professoren. Deshalb werden die Kenntnisse des Lehrpersonals, der Wissenschaftler und Forscher verbessert. Eine weitere Aufgabe betrifft Publikationen und die Gewinnung langfristiger wissenschaftlicher Ergebnisse. Auch die Kapazitäten für internationale Zusammenarbeit, der Bibliothek, an Lehr-, Forschungs- und administrativem Personal, Anlagen und Gebäuden sollen vergrößert werden.

Andere Aufgaben betreffen öffentliche Informationen über Forschungsergebnisse, Beratung von Firmen, der Zentralregierung und von Gemeinden, die Wirtschaftsförderung, die Unterstützung von Forschungszentren, z. B. das Zentrum für „Entrepreneurship“, die Unterstützung der Colleges und die Einwerbung europäischer Gelder. Die Universität bietet ihre Kapazitäten zur politischen und öffentlichen Entscheidungsfindung an und soll Einkommenserhöhungen und die Wirtschaftsentwicklung in Südestland fördern. Über die Colleges erfolgt dies auch in anderen Regionen Estlands.

Die Erfüllung dieser Aufgaben signalisiert weitgehend soziale Erfolge (soziale Nutzen), während Fehlschläge bei der Aufgabenerfüllung mit sozialen Misserfolgen (soziale Kosten) einhergehen. Beispiele bilden Studienabbrüche oder nicht bestandene Examen in den Bachelor- und Masterstudiengängen. soziale Kosten signalisieren abgebrochene Doktorandenstudien, der Zusammenoder Abbruch von Forschungsprojekten, nicht beendete internationale Projekte, Unfälle, Emissionen, die Abwanderung von Personal, erfolglose Beratung, Ressourcenverluste infolge persönlicher Beratungstätigkeit, die Blockierung von Ressourcen für andere Zwecke, politische Aktivitäten, und resultierende Belastungen der Standortregion.

Demnach besitzen die Aktivitäten der Fakultät zahlreiche soziale Implikationen. Als Wirtschaftseinheit nutzt die Fakultät Bestände von Produktionsfaktoren in Form von Vermögen, z. B. Standort, Gebäude, Anlagen, Parkplätze. Ebenso erfolgen laufende Faktoreinsätze, die Strömungsgrößen darstellen, aber für andere Verwendungen nicht mehr zur Verfügung stehen. Andererseits wurden Kapazitäten aufgebaut, um Erkenntnisse zu gewinnen und um bessere soziale Verhältnisse zu schaffen. Sozialen Wirkungen besitzen sowohl Bestands- als auch Strömungsgrößen.

Die oben erwähnten Aufgaben erfordern laufende Aktivitäten. Soziale Effekte betreffen Studenten und andere Wirtschaftseinheiten wie Unternehmen, öffentliche Verwaltungen Gebietskörperschaften und Forschungsinstitute. Manchmal verändern soziale Effekte Bestände. Öfters treten 
sie nur während einer Periode auf und beeinflussen den sozialen Erfolg nur in dieser Periode als Strömungsgrößen.

Um die Effekte und somit soziale Erfolge arbeitsteiligen Wirtschaftseinheiten zuzurechnen, sind Managementinstrumente, z. B. im Rahmen eines Rechnungswesens erforderlich, um die sozialen Erfolge bzw. Misserfolge zu messen (Bräuning/ Eichhorn 2002).

\section{Der Kontenrahmen und die Buchführungsregeln}

Die kommerzielle Rechnung (siehe Abbildung 1) wird zunächst für die Fakultät erstellt. Das vorhandene Buchführungssystem der Fakultät ist bezüglich der Budgetstruktur und der Buchführung gemäß den Buchführungsgesetzen, die für Verwaltungen und öffentlichen Unternehmen Estlands gelten, ausgestaltet (vgl. University of Tartu 2006; ähnlich Heiling 2007, S. 129). Einnahmen und Ausgaben werden in Konten gebucht, die der Erfolgsrechnung dienen, und in Bestandskonten für Vermögens- und Verbindlichkeitsänderungen. Weiterhin existieren einige Konten der Gesamtuniversität für die Vermögen, wie Grundstücke, Lagerbestände, Kundenvorauszahlungen, das Eigenkapital, Reserven und Verbindlichkeiten, Rechnungsabgrenzungen (Department of Bookkeeping 2006). Aufspaltungen solcher Konten sind für die Fakultät und die mit ihnen verbundenen Colleges erforderlich. ${ }^{8}$ Einnahmen (Erträge), Ausgaben (Aufwendungen), Vermögen, Verbindlichkeiten und das Kapital der Fakultät wurden für das Jahr 2006 erfasst, gebucht, der kaufmännische Erfolg ermittelt und eine kommerzielle Schlussbilanz der Fakultät erstellt. Der resultierende Verlust in der kommerziellen Erfolgsrechnung wurde von der Universität kompensiert und in Tabelle 6 vom Kapital abgezogen. Diese Schlussbilanz wurde in die soziale Gesamtbilanz überführt.

Im Anschluss wendeten wir uns den zusätzlichen sozialen Buchungen (siehe Abbildung 1) zu. Strömungsgrößen werden in zusätzlichen laufenden sozialen Nutzenkonten und in zusätzlichen ${ }^{9}$ sozialen laufenden sozialen Kostenkonten gebucht (vgl. Abbildung 1). Gegenbuchungen erfolgen in dem Konto zusätzliche soziale Kasse. Besondere Abgrenzungskonten dienen der Bestimmung jener zusätzlichen sozialen Nutzen und sozialen Kosten, die nicht von den Aktivitäten der Fakultät herrühren.

Bestände werden als soziale Vermögen und soziale Verbindlichkeiten einbezogen. Die sozialen Vermögen bezeichnen soziale Nutzen, die länger als eine Periode vorhanden sind. Soziale Verbindlichkeiten umfassen soziale Kosten, die ebenfalls eine Periode überdauern. Die Bestandskonten zeigen längerfristig dauernde soziale Nutzen und längerfristig bestehende soziale Kosten (Verbindlichkeiten) auf. Wiederum werden Abgrenzungskonten für diese Bestände benötig (vgl. Abbildung 1 und Tabelle 5).

8 Einige Grundstücke, welche die Colleges nutzen, z. B. in Pärnu, werden von anderen Gebietskörperschaften zur Verfügung gestellt Infolge der Bewertungstechniken, die in Estland im öffentlich kaufmännischen Rechnungswesen angewendet werden, beeinflussen Neubewertungen der Grundstücke den Gewinn, die Reserven und das eigene Kapital.

9 Aus Gründen der besseren Lesbarkeit wird das Wort zusätzlich im folgenden Text nicht mehr verwendet; alle soziale Nutzen und soziale Kosten sind „zusätzlich“, da ein Teil von ihnen schon im kaufmännischen Rechnungswesen erfasst worden sind. 
Das Kontensystem illustrieren die Abbildung 1 und die Tabellen 3,4 und 5, die den Kontenplan für die sozialen Konten verdeutlichen. Er ist gemäß den Wirkungen der Fakultätsaktivitäten und den sozialen Eigenheiten der zu buchenden Transaktionen aufgebaut. Die Bezeichnung II bezieht sich auf ein Konto im zusätzlichen sozialen Rechnungswesen. ${ }^{10}$ Der Buchstabe F verdeutlicht die Fakultät.

\begin{tabular}{|c|c|c|}
\hline Vermögen & $\begin{array}{c}\text { Klasse } \\
\text { ، } \\
\text { “ }\end{array}$ & $\begin{array}{l}\text { 0: Materielle soziale Vermögen, } \\
\text { soziales Humanvermögen } \\
\text { 1: soziale Kasse } \\
\text { 2: Soziale Forderungen }\end{array}$ \\
\hline Verbindlichkeiten & “ & $\begin{array}{l}\text { 3:soziales Eigenkapital, } \\
\text { Wertberichtigungen } \\
\text { 4: soziale Verbindlichkeiten und } \\
\text { soziale Netto-Nutzen }\end{array}$ \\
\hline Soziale Nutzen & “" & 5: soziale Nutzen \\
\hline Soziale Kosten & “ & $\begin{array}{l}\text { 6: soziale Material- und } \\
\text { Personalkosten }\end{array}$ \\
\hline $\begin{array}{l}\text { Technische } \\
\text { Konten }\end{array}$ & “ & $\begin{array}{l}\text { 7: soziale Eröffnungsbilanz, Soziale } \\
\text { Endbilanz, soziale Gesamtbilanz, } \\
\text { soziales Erfolgskonto } \\
\text { 8: Abgrenzung Bestände } \\
\text { 9: Abgrenzung Erfolge }\end{array}$ \\
\hline
\end{tabular}

\section{Tabelle 3: Gruppen der zusätzlichen sozialen Konten}

Quelle: Darstellung der Autoren

Der Kontenplan und die Konten in den Tabellen 4 bis 5 berücksichtigen die Eigenheiten der Fakultät, die in Kapitel III angesprochen worden sind. Diese Konten mussten bestimmt werden. Sie sollen die Beziehungen zwischen den Aktivitäten der Fakultät und sozialen Nutzen und sozialen Kosten widerspiegeln, wobei die Intensität dieser Zusammenhänge - dargestellt mittels einer Matrix - von den Eigenheiten der zur Aufgabenerledigung erforderlichen Transaktionen und von der grundsätzlichen Bewertungsmethode mittels des Zahlungsbereitschaftsansatzes der

10 Die Klassifikationsnummern 0 bis 2 kennzeichnen soziale Vermögen, während die sozialen Verbindlichkeiten die Klassifikationsnummern 3 bis 4 aufweisen. Laufende soziale Nutzen sowie soziale Kosten werden in der Kontengruppe 5 und 6 gebucht. Die technischen Konten umfassen die soziale Eröffnungsbilanz und die soziale Schlussbilanz, das soziale Erfolgskonto und die gesamte soziale Bilanz in der Kontengruppe 7. Die Klassifikationsnummer 8 betrifft die Abgrenzung der sozialen Bestände und die Gruppe 9 die Abgrenzung laufender sozialer Transaktionen. 
Nutzen-Kosten- Analyse abhängt. Es wurden Intensitätsstufen eingeführt, die erlauben, die wichtigsten Beziehungen zu erkennen. Wir konzentrierten uns auf jene Beziehungen, die besonders intensiv sind, um sie als Basis für die Kontenarten zu verwenden.

Es resultieren die einzelnen Bestandskonten der sozialen Vermögen und sozialen Verbindlichkeiten. Hier dominiert vor allen die Bestandsart die erwähnten Beziehungen, während die laufenden sozialen Nutzen und laufenden sozialen Kosten von den Aufgabenarten geprägt werden. Soziale Vermögen (vgl. Abbildung 1) resultieren in erster Linie aus:

Verbesserungen des Wissensstandes und der Fertigkeiten zu forschen und zu lehren, Infrastrukturvergrößerungen, Chancen für Einnahmenerhöhungen, zusätzlichen Beschäftigungschancen, Forschungsergebnissen, erweiterten Forschungs- und Lehrkapazitäten und Beratungsmöglichkeiten.

Weitere soziale Werte, die im kommerziellen Rechnungswesen der Fakultät nicht ausgewiesen werden, betreffen Anlagen, Gebäude sowie Einrichtungen, Lagerbestände sowie das kommerzielle Reinvermögen der Fakultät. Zudem wird das soziale Kasse Konto für die Aufnahme der Gegenbuchungen der sozialen Nutzen und Kosten ausgewiesen.

Ferner existieren soziale Wertberichtigungskonten für die sozialen Vermögen das Fakultätsgebäude und andere Vermögen. Hinzu kommen Wertberichtungen für die sozialen Verbindlichkeiten. Zu letzteren zählen Berichtigungen von Unfallfolgen, die Fakultät betreffende Verschuldungen, Wertänderungen bei Grundstücken, soziale Verluste infolge der Zuweisungen an die Fakultät. Für Verbindlichkeiten in Zusammenhang mit Gebäuden und für zusätzliche Nettonutzen werden Konten geführt.

Konten für soziale Nutzen folgen aus den Aktivitäten der Fakultät sowie der drei Colleges zur Erledigung ihrer Hauptaufgaben. Sie betreffen fünf Gruppen (vgl. Tabelle 4) Lehre (1), Forschung (2), Beratung (3), Management (4) und andere Aufgaben (5):

Lehre (1): Hier werden Aufgaben erfasst, wie die Belehrung von Bachelor- und Masterstudenten, berufsbezogene Ausbildung, Fernunterricht, Unterrichten im Rahmen gemeinsamer Projekte mit der Wirtschaftspraxis, Doktorandenausbildung, Wochenendausbildung (Open University), Examina, Verfassen von Lehrmaterial und die Anfertigung von Lehrbüchern.

Forschung (2): Im Rahmen der Forschung findet statt: die Weiterbildung von Wissenschaftlern, die Veröffentlichung von Forschungsergebnissen, die Verbreitung von Informationen, wissenschaftliche Beratungen, Einwerben von Geldern, Verfassung von Büchern, Forschungsberichten, Organisation von Konferenzen und anderen wissenschaftlichen Treffen.

Beratung (3): Beratungen erfolgen für Firmen, für öffentliche Körperschaften, und Verwaltungen. Sie betreffen die Regionalplanung, die Mitwirkung im estnischen Parlament, die Tätigkeit von Kammern, von Vorständen, Aufsichtsräten, die Europäische Union, und die Arbeit wissenschaftlicher Gremien. Management (4): Managementaufgaben betreffen Kontrollaktivitäten, die Bewirtschaftung von Geldern, Personalführung, Entscheidungen und Planungen der Fakultät, Mitwirkung bei Universitätsentscheidungen, die Führung von Rechenzentren und Abteilungen für spezielle Aufgaben, Kontakte zu anderen Fakultäten, die Unterstützung der Colleges und die Repräsentation der Fakultät.

Andere Aufgaben (5): Sie haben Kontakte zu Schulen, die Unterstützung des Besucherverkehrs, Public Relations, Werbung, usw. zum Gegenstand.

Da die sozialen Nutzen mit Hilfe des Zahlungsbereitschaftsansatzes bestimmt werden, reflektieren die Einnahmen (Erträge) der Fakultät einen Teil der sozialen Nutzen. Die Zahlungsbereitschaft äußert sich in den Zuweisungen der Zentralregierung, des Estnischen Forschungsfonds, und in Zahlungen anderer Institutionen, etwa von Gemeinden, Unternehmen Eltern, den Verwandten von 
Studenten sowie in den Zahlungen der Studenten selbst. Die Zahlungen der Institutionen drücken die mittelbaren Wünsche der Entscheidenden im Namen einer Gruppe aus, ${ }^{11}$ z. B. in den Zuweisungen der Zentralregierung und in den Zahlungen anderer Fakultäten. Die Einnahmen aus diesen Zahlungen an die Fakultät werden im kaufmännischen Rechnungswesen erfasst. Deshalb konzentrieren sich die sozialen Nutzen-Konten auf die Zahlungsbereitschaft für jene Effekte, die nicht im kommerziellen Rechnungswesen Beachtung erfahren.

Neben den reinen Finanzvorgängen weisen viele der Aktivitäten zur Realisierung der Aufgaben (1) bis (3) erzieherische und externe Effekte auf, die zur Einrichtung von Konten für soziale Nutzen Anlass geben. Andere soziale Nutzen rühren vom Management und den Investitionen der Fakultät her. Diese sozialen Nutzen werden auf Konten, die sich auf die Aufgabengruppe (4) und (5) beziehen, erfasst. Die Wirkungen, die zu sozialen Nutzen führen, treten bei Haushalten, z. B. Studenten und deren Angehörigen, Unternehmen in etlichen Sektoren, Infrastruktureinrichtungen, Versicherungen, Verwaltungen und anderen öffentlichen Wirtschaftseinheiten auf. Die Steuereinnahmen der Standortgemeinde und soziale Nutzen aus indirekten Steuern werden ebenfalls einbezogen.

Soziale Kosten bewirken die oben aufgeführten Aktivitäten der Fakultät. Sie bezeichnen soziale Kosten der Finanzierung, Forschung, Lehre usw. Diese sozialen Kosten verdeutlichen die Zahlungsbereitschaft für den Einsatz von Produktionsfaktoren, d. h. deren anderweitige Verwendung. Sie gelangen teilweise in den Ausgaben (Aufwendungen) der Fakultät und im existierenden kaufmännischen Rechnungswesen zum Ausdruck. Das kommerzielle Rechnungswesen verweist ferner auf Verluste, die vom öffentlichen Träger (Eigentümer) gedeckt werden sowie auf die $\mathrm{Ab}$ schreibungen auf Gebäude und Einrichtungen. Zahlungsbereitschaftsansätze müssen bemüht werden, um soziale Abschreibungen auf Wissen, soziale Kosten von Unfällen, zusätzliche Reisekosten und Zeitverluste zu monetisieren, Sponsoring führt ebenfalls zu sozialen Nutzen und Kosten. Einige Kosten, die im kaufmännischen Rechnungswesen nicht erfasst werden, sind ebenfalls einzubeziehen. Dazu zählen Abschreibungen auf unbewegliche und bewegliche Vermögen, die sich im Eigentum anderer öffentlicher Träger befinden.

Diese Eigenheiten verdeutlichen die Kontennummern ${ }^{12}$ in den Tabellen 4 bis 6, wo die soziale Bilanz, die soziale Erfolgsrechnung und die soziale Gesamtbilanz dargestellt werden.

Die Bewertungen der einzelnen laufenden Transaktionen erfolgt gemäß dem Grundansatz. Die realisierten Zahlungsbereitschaften finden sich im kaufmännischen Rechnungswesen, z. B. die Zahlungen der Bachelorstudenten (Einnahmen der Fakultät) als kaufmännisch erfasste soziale

11 Z. B. in den Zuweisungen der Zentralregierung und in den Zahlungen anderer Fakultäten. Ebenfalls mittelbare Zahlungsbereitschaften signalisieren die Zahlungen für Dienstleistungen seitens anderer Institutionen, z. B. der Handelskammern, Personenkörperschaften, von Vereinen und Vereinigungen. Die meisten dieser Institutionen sind mitgliedschaftlich organisiert, so dass deren Zahlungen Mitgliederwünschen bezüglich der Aufgabenerfüllung dieser Institutionen zum Ausdruck bringen.

12 Die erste Zahl einer Kontonummer weist auf die Kontenart, z. B. in Tabelle 2 und 4, hin. Der Buchstabe A symbolisiert soziale Vermögen, während der Buchstabe L soziale Verpflichtungen kennzeichnet. B bezeichnet zusätzliche soziale Nutzen und sC zusätzliche soziale Kosten, während V Wertberichtungen der zusätzlichen sozialen Vermögen, W Wertberichtungen zusätzlicher sozialer Verpflichtungen und T auf technische Konten verweisen. Die zweite Zahl bezieht sich auf eine Art zusätzlicher sozialer Nutzen und Kosten, während die dritte Zahl eine Untergruppierung dieser zusätzlichen sozialen Nutzen und Kosten oder auf solche zusätzlichen sozialen Nutzen und Kosten verweist, die aus speziellen Fakultätsaktivitäten resultieren. Die vierte Stelle der Kontonummer weist eine 1 auf, falls keine Ausgrenzung erforderlich wird. Auf Ausgrenzungsnotwendigkeiten deutet hingegen die Nummer 2 hin. 
Nutzen. Als zusätzliche soziale Nutzen erscheinen die Konsumentenrenten, der Bachelorstudenten, die in der Fakultät verbleiben. Ihre Größe nimmt den Wert (Konto B5101) 4,858 Mill. Kronen an. ${ }^{13}$ Auch die Ausgaben für Bachelorstudenten erscheinen im kaufmännischen Rechnungswesen als soziale Kosten. Als zusätzliche Zahlungen der Studenten für Lebenshaltungskosten, die nicht durch deren Einkommen gedeckt sind, wurden 1,822 Mill. Kronen (Konto sC6101) als zusätzliche soziale Kosten errechnet. (z. B. Zuschüsse von Eltern). Unter Anwendung der oben (Kapitel II, 1) erwähnten Bewertungsverfahren und unter Berücksichtigung der erreichbaren Daten wurden die laufenden sozialen Nutzen und laufenden sozialen Kosten bestimmt.

Die sozialen Vermögen bestehen beispielsweise aus den im kaufmännischen Rechnungswesen der Fakultät nicht gebuchten Wert von Büchern der Fakultätsbibliothek und der Zentralbibliothek $^{14}$ sowie eines Ansatzes für den Humanwert des Fakultätspersonals ${ }^{15}$ (Konto IIFA011) in Höhe von 36,328 Mill. Kronen. Soziale Schulden werden beispielsweise von Emissionen in Höhe von 0,710 Mill. Kronen bewirkt. Hier rekurrieren wir auf die Emissionen der Kraftfahrzeuge, die zum Erreichen der Fakultät benutzt werden. ${ }^{16}$ Mittels der indirekten Bewertungsmethoden werden die anderen Wertbestände ebenfalls ermittelt. Für den jährlichen Abbau des sozialen Vermögens und der sozialen Schulden werden Wertberichtigungen vorgenommen, die die einzelnen Vermögen und Schuldenbestände betreffen und in den Wertberichtigungskonten für Passiva (Konten IIFW3001- 3511) in Höhe von 0,147 Mill. Kronen und für Aktiva in Höhe von 10,170 Mill. Kronen (Konten IIFV3101-3612) - in Tabelle 6 gesammelt - ausgewiesen werden. ${ }^{17}$

Zu Beginn einer Periode benutzen wir die gesamte soziale Bilanz der letzten Periode, die zugrunde liegende soziale Bilanz und die kommerzielle Schlussbilanz. Transaktionen werden mit ihren kaufmännischen Konsequenzen im kommerziellen Buchführungssystem erfasst. Die Buchung sozialer Werte erfolgt in den Konten für soziale Nutzen und Kosten. Nach Abschluss dieser Konten werden deren Salden sowie die sozialen Abschreibungen auf das Konto soziale Erfolgsrechnung übertragen (vgl. Abbildung 1,Tabelle 4).

13 Für die Konsumentenrente wurde das Näherungsmaß von Marshall herangezogen, wobei mit Hilfe des Dekans eine Prohibitivgebühr, bei der die Nachfrage für das Bachelorstudium verschwindet, geschätzt wurde. Von dieser wurde die tatsächliche Gebühr pro Student abgezogen und durch zwei dividiert und mit der Zahl der betreffenden Studenten multipliziert.

14 Schätzungen der Bibliothekare der Fakultät und der Zentralbibliothek.

15 Es wurde angenommen, dass das ersetzendes Personal im Jahre 2006 etwa $20 \%$ teurer gewesen wäre.

16 Aus der Literatur wird ein Schadenswert pro Tonne CO2 entnommen. Pro gefahrenen Kilometer entstehen 251 g CO2 diese Menge wird mit der Zahl der gefahrenen Kilometer des Personals, der Studenten, Gäste usw. multipliziert, die Gesamtmenge errechnet und der entstandene Schadenswert bestimmt.

17 Die Wertberichtigungssätze wurden aus Studien zum Wissensverlust, z. B. $10 \%$ bei Doktoranden, dem Emmissionsschadensabbau usw. entnommen und geschätzt. 

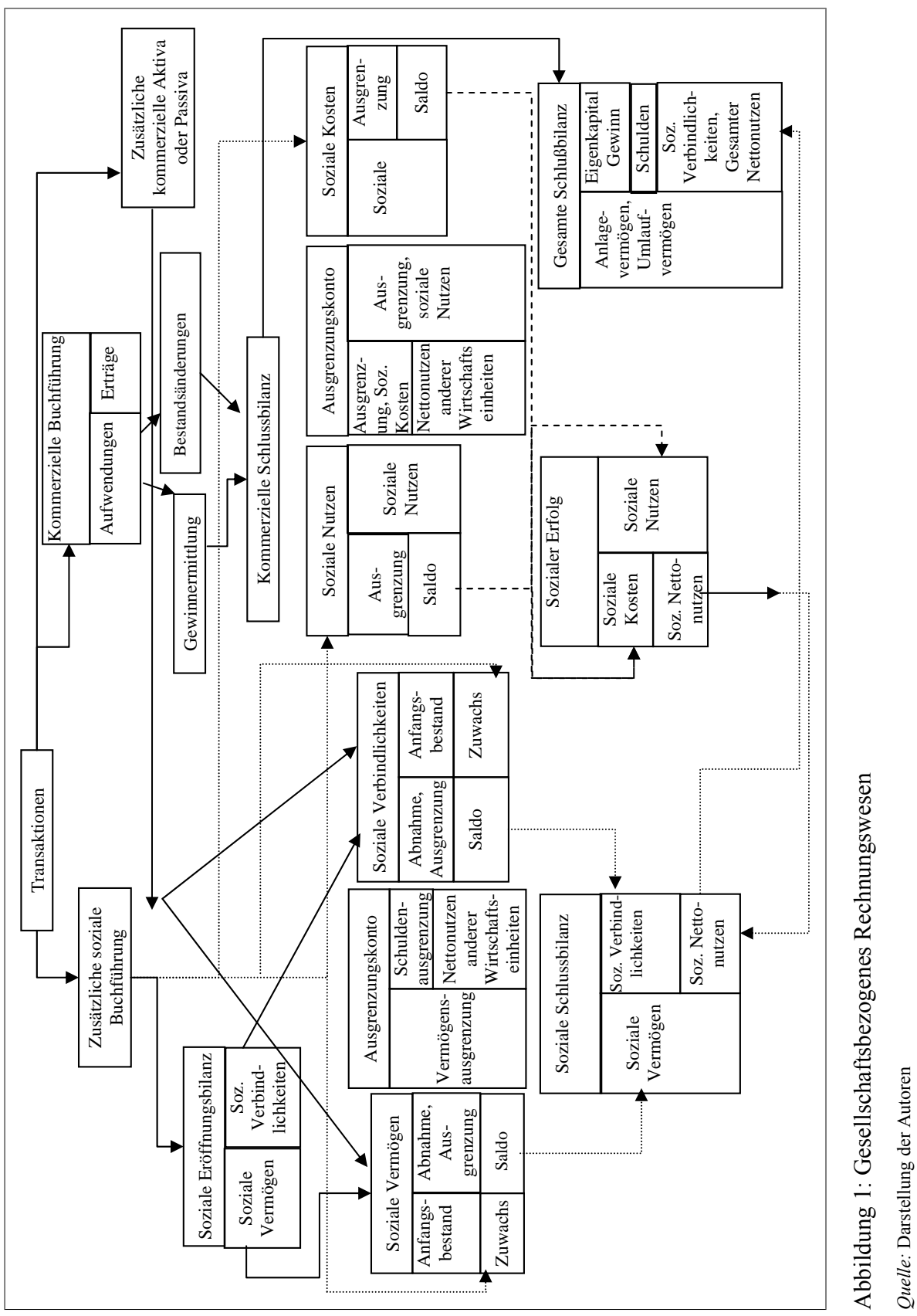


\begin{tabular}{|c|c|c|c|c|c|}
\hline \multirow{2}{*}{$\begin{array}{l}\text { IIFT7201 } \\
\text { Lehre (1) }\end{array}$} & \multicolumn{2}{|c|}{ Soziale Kosten aus Mill. Kronen } & \multicolumn{3}{|c|}{ Soziale Nutzen aus Mill. Kronen } \\
\hline & & & & & \\
\hline sC6101 & Bachelor Studien & 1,822 & B5101 & Bachelor Studien & 4,858 \\
\hline sC6111 & Master Studien & 0,018 & B5111 & Master Studien & 2,418 \\
\hline sC6122 & Doktor Studien & 0,183 & B5122 & Doktor Studien & 5,547 \\
\hline sC6132 & Weiterbildung & 0,324 & B5132 & Weiterbildung & 0,513 \\
\hline sC6141 & Open University & 0,928 & B5141 & Open University & 11,775 \\
\hline sC6152 & Veröff. Lehrmaterial & 0,048 & B5152 & Veröff. Lehrmaterial & 0,924 \\
\hline \multicolumn{6}{|c|}{ Forschung (2) } \\
\hline sC6202 & Veröff. Forschungsresultate & 0,004 & B5202 & Veröff. Forschungsresultate & 0,008 \\
\hline sC6212 & Gelder Einwerbung & 0,298 & B5212 & Gelder Einwerbung & 0,658 \\
\hline sC6222 & Forschungsanträge & 0,365 & B5222 & Forschungsanträge & 0,609 \\
\hline sC6231 & Anfertigung Artikel, Bücher & 0,496 & B5231 & Anfertigung Artikel, Bücher & 1,548 \\
\hline sC6242 & Konferenzorganisation & 0,139 & B5242 & Konferenzorganisation & 0,148 \\
\hline \multicolumn{6}{|c|}{ Beratung (3) } \\
\hline sC6301 & Unternehmen & 0,636 & B5301 & Unternehmen & 1,060 \\
\hline sC6311 & Öffentliche Verwaltungen & 0,541 & B5311 & Öffentliche Verwaltungen & 1,351 \\
\hline sC6321 & Parlament & 0,030 & B5321 & Parlament & 0,360 \\
\hline sC6331 & $\mathrm{EU}$ & 0,019 & B5331 & $\mathrm{EU}$ & 0,060 \\
\hline sC5341 & Wissensch. Institutionen & 0,023 & B5341 & Wissensch. Institutionen & 0,046 \\
\hline \multicolumn{6}{|c|}{ Management (4) } \\
\hline sC6401 & Kontrolle & 0,015 & B5401 & Kontrolle & 0,231 \\
\hline sC6411 & Finanzielles Management & 0,015 & B5411 & Finanzielles Management & 0,219 \\
\hline sC6421 & Personalmanagement & 0,024 & B5421 & Personalmanagement & 0,285 \\
\hline sC6431 & Fakultätsentscheidungen & 0,043 & B5431 & Fakultätsentscheidungen & 0,642 \\
\hline sC6441 & Management Laboratorien & 0,003 & B5441 & Management Laboratorien & 0,041 \\
\hline sC6452 & Kontakte andere Fakultäten & 0,002 & B5452 & Kontakte andere Fakultäten & 0,027 \\
\hline sC6461 & Unterstützung Colleges & 0,076 & B5461 & Unterstützung Colleges & 0 \\
\hline sC6471 & Repräsentation & 0,012 & B5471 & Repräsentation & 0,024 \\
\hline \multicolumn{6}{|c|}{ Andere Fakultätsaktivitäten (5) } \\
\hline sC6501 & Schulkontakte & 0,002 & B5501 & Schulkontakte & 0,003 \\
\hline sC6512 & Besucher und Gäste & 0,154 & B5512 & Besucher und Gäste & 0,456 \\
\hline sC6522 & Public relations & 0,001 & B5522 & Public Relations & 0,840 \\
\hline sC6532 & Werbung & 0,004 & B5532 & Werbung & 0,108 \\
\hline sC5542 & Fiskalische soz. Kosten & 0 & B5542 & Fiskalische soz. Nutzen & 6,773 \\
\hline sC6552 & Andere soz. Kosten & 0,200 & B5552 & Andere soz. Kosten & 0 \\
\hline sC6701 & Abschreibung & 10,170 & B5801 & Zuschreibung & 0,147 \\
\hline \multirow[t]{2}{*}{ B4871 } & Zus. Soz. Nettonutzen & 25,84 & & & \\
\hline & & 41,679 & & & 41,679 \\
\hline
\end{tabular}

Tabelle 4: Zusätzliches Erfolgskonto der Fakultät

Quelle: Darstellung der Autoren 
Ein Kontenrahmen für eine "gesellschaftsbezogene Rechnungslegung"

\begin{tabular}{|l|l|}
\hline T7101 & Soziale Eröffnungsbilanz \\
\hline T7201 & Soziales Erfolgskonto \\
\hline T7301 & Soziale Bilanz \\
\hline T7401 & Gesamte soziale Bilanz \\
\hline T8202 & Ausgrenzungen von soz. Vermögen (Bestände) \\
\hline T8212 & Ausgrenzungen von soz. Verbindlichkeiten (Bestände) \\
\hline T9302 & Ausgrenzung von soz. Nutzen \\
\hline T9312 & Ausgrenzung von soz. Kosten \\
\hline
\end{tabular}

\section{Tabelle 5: Technische Konten}

Quelle: Darstellung der Autoren

Das Konto zusätzliche soziale Erfolgsrechnung wird abgeschlossen und der Saldo in die soziale Schlussbilanz übertragen. Dort erscheinen die Salden der sozialen Vermögen, die soziale Kasse, die sozialen Verbindlichkeiten sowie die Wertberichtigungen sozialer Vermögen und Verbindlichkeiten (vgl. Tabelle 6). In einem weiteren Schritt wird die soziale Schlussbilanz mit der kommerziellen Schlussbilanz zur gesamten sozialen Schlussbilanz zusammengefasst.

\begin{tabular}{|c|c|c|c|c|c|}
\hline \multirow{3}{*}{$\begin{array}{c}\text { IIFT7401 } \\
\text { IFT711 } \\
\text { IFT711 }\end{array}$} & \multicolumn{5}{|c|}{ Gesamte soziale Bilanz (Mill. Kronen) } \\
\hline & \multicolumn{5}{|c|}{ Kommerzielle Bilanz } \\
\hline & \multicolumn{2}{|l|}{ Kommerzielle Vermögen } & \multirow{2}{*}{$\begin{array}{c}\text { IFT711 } \\
1 .\end{array}$} & \multicolumn{2}{|l|}{ Kommerzielle Verbindlichkeiten } \\
\hline 1. & Anlagevermögen & 71,515 & & Nettovermögen, Kapital & 72,714 \\
\hline 2. & Beteiligungen & 0 & 2. & Verbindlichkeiten & 3,023 \\
\hline 3. & Umlaufvermögen & 3,222 & & & \\
\hline 4. & Abgrenzungen & 1,0 & & & \\
\hline IIFT7301 & \multicolumn{5}{|c|}{ Zusätzliche soziale Bilanz } \\
\hline IIFT7301 & Zus. Soziale Vermögen & 0 & IIFT7301 & Zus. Soziale Verbindlichkeiten & 0 \\
\hline IIFA0101 & Wert von Gebäuden & 0 & IIFL4001 & $\begin{array}{l}\text { Vorherige } \\
\text { Nettonutzenbestände }\end{array}$ & 0 \\
\hline IIFA0111 & Vermögen nicht gebucht & 36,328 & IIFL4011 & Unfälle & 0 \\
\hline IIFA0201 & $\begin{array}{l}\text { Wissen } \\
\text { Bachelorstudenten }\end{array}$ & 3,238 & IIFL4021 & Emissionen etc. & 0,710 \\
\hline IIFA0211 & Wissen Masterstudenten & 1,093 & IIFL4101 & Zuk. finanzielle Belastungen & 0 \\
\hline IIFA0222 & Wissen Doktoren & 0,544 & IIFL4201 & Aufgabe von consultancy & 0 \\
\hline IIFA0231 & Wissen Open University & 6,615 & IIFL4301 & Personalverluste & 0 \\
\hline IIFA0242 & Wissen Weiterbildung & 0,354 & IIFL4401 & Engagement Politik & 0,020 \\
\hline IIFA0251 & Wissen Lehrkörper & 0,031 & IIFL4501 & Beschäftigungsverluste & 0 \\
\hline IIFA0261 & Wissen Forscher & 0,030 & IIFL4511 & Verringerung Infrastruktur & 0 \\
\hline IIFA0301 & $\begin{array}{l}\text { Dauernde } \\
\text { Forschungsresultate }\end{array}$ & 11,400 & IIFL1001 & $\begin{array}{l}\text { Soz. Kasse } \\
\text { (zusätzliches Soz. Kapital) }\end{array}$ & 42,387 \\
\hline
\end{tabular}




\begin{tabular}{|c|c|c|c|c|c|}
\hline IIFA0312 & $\begin{array}{l}\text { Erhöhung. intern. } \\
\text { Kooperationskap. }\end{array}$ & 0,603 & $\begin{array}{l}\text { IIFV3101- } \\
3612\end{array}$ & Wertberichtigungen $^{18}$ & 10,170 \\
\hline IIFA0321 & $\begin{array}{l}\text { Erh. Forschungs- } \\
\text { personalkapazität }\end{array}$ & 1,426 & & & \\
\hline IIFA0331 & $\begin{array}{l}\text { Erh. Forschungs- } \\
\text { ausrüstungskap. }\end{array}$ & 0,160 & & & \\
\hline IIFA0341 & $\begin{array}{l}\text { Erh. Forschungs- } \\
\text { gebäudekap. }\end{array}$ & & & & \\
\hline IIFA0351 & $\begin{array}{l}\text { Erh. Forschungsbestand } \\
\text { Bücher }\end{array}$ & 0,094 & & & \\
\hline IIFA0361 & $\begin{array}{l}\text { Beiträge zu Forschungs- } \\
\text { zentren }\end{array}$ & 0 & & & \\
\hline IIFA0401 & Beratungskapazitäten & 1,060 & & & \\
\hline IIFA0411 & $\begin{array}{l}\text { Kapaz. zur Regierungs- } \\
\text { beratung }\end{array}$ & 0,901 & & & \\
\hline IIFA0421 & $\begin{array}{l}\text { Europäische Fonds- } \\
\text { mittel }\end{array}$ & 0,060 & & & \\
\hline IIFA0501 & Entw. Estnische Sprache & 1,915 & & & \\
\hline IIFA0511 & $\begin{array}{l}\text { Beschäftigungs- } \\
\text { erhöhung }\end{array}$ & 2,724 & & & \\
\hline IIFA0521 & $\begin{array}{l}\text { Infrastruktur- } \\
\text { erweiterung }\end{array}$ & 3,500 & & & \\
\hline IIFA0601 & $\begin{array}{l}\text { Gewinne anderer } \\
\text { Unternehmen }\end{array}$ & 1,554 & & & \\
\hline IIFA0612 & $\begin{array}{l}\text { Erhöhte } \\
\text { Steuereinnahmen }\end{array}$ & 4,594 & & & \\
\hline \multirow{3}{*}{$\begin{array}{l}\text { IIFW3001- } \\
3511\end{array}$} & Wertberichtigungen & 0,147 & & & \\
\hline & & & IIFB4871 & Zu. Nettonutzen & 25,084 \\
\hline & & 154,108 & & & 154,108 \\
\hline
\end{tabular}

Tabelle 6: Gesamte soziale Bilanz (Kommerzielle und zusätzliche soziale Bilanz)

Quelle: Darstellung der Autoren

Der gesamte soziale Nettonutzen fällt für die Fakultät positiv aus, obwohl sich der kaufmännische Verlust auf 2 Mill. Kronen (127.823 Euro) beläuft. Der zusätzliche soziale Nutzen ist beträchtlich höher als dieser Verlust. Da die Fakultät eine Non-Profit-Institution darstellt, hängt der zusätzliche soziale Nettonutzen von dem zusätzlichen sozialen Nutzen und Kosten, insbesondere der Forschung und der Lehre ab. Der soziale Nettonutzen beläuft sich auf 25 Mill. Kronen (ca. 1,6 Mill. Euro). Ferner existiert ein zusätzliches soziales Kapital von 42.4 Mill. Kronen (ca. 2,7 Mill. Euro). Das gesamte Cluster bestehend aus Fakultät und den Colleges weist einen sozialen Nettonutzen von 38.2 Mill. (ca. 2,4 Mill. Euro) und ein zusätzliches soziales Kapital von 96.5 Mill. Kronen (ca.6, 2 Mill. Euro) auf.

18 Die Wertberichtungen IIFV3101-3612 resultieren aus der Summe der einzelnen Wertberichtigungen der einzelnen soziale Vermögen, die die Abnahme dieser Vermögen in der betrachteten Periode aufzeigen, z. B. für den Wissensbestand der Doktoranden in Höhe von 10\%. Die Wertberichtungen IIFW3001-3511 reflektieren die Summe der Abnahme sozialer Verbindlichkeiten. 


\section{Probleme eines sozialen Rechnungswesens für eine Fakultät}

Die Argumente gegen und für die Verwendung eines solchen Rechnungswesens beziehen sich grundsätzlich auf ein solches Rechnungswesen oder ein solches Rechnungswesen, das auf eine Fakultät ausgerichtet ist. Die erste Gruppe von Argumenten lautet:

1. Die Basis für ein solches Rechnungswesen bildet die individualistische Wohlfahrtstheorie (van Graaff 1963; Ahlheim/ Rose 1989), deren Wertermittlung nicht unbedingt die wirkliche Werthaltung einer Gesellschaft widerspiegelt. Die Rolle von sozialen Gruppen wie Priesterschaften, Funktionäre, Militärs, Beamten, Gewerkschaften, Parteien und von Parlamenten sowie von Gerichten bei der Bestimmung der gesellschaftlichen Wohlfahrt wird ignoriert. Darüber hinaus werden anspruchsvolle Annahmen über die Gleichheit der Zahlungsbereitschaft, die sich in einer Krone (nun Euro) ausdrückt, formuliert. Man ignoriert die Tatsache, dass ein Euro für eine reiche Person eine andere Bedeutung besitzt als für eine arme Person. Die so genannten Kompensationstests als Basis für die Differenz zwischen sozialen Nutzen und sozialen Kosten werden in der Wohlfahrtsliteratur konträr diskutiert.

2. Sowohl der Tausch als auch die Koordination sozialer und ökonomischer Güter erfolgt nicht nur monetär über Märkte, z. B. mittels horizontaler und vertikaler Transfers von Realgütern und Geld, der Befehlsgebung, externer Effekte. Obwohl diese Schwierigkeiten bestehen, ist die Nutzen-Kosten-Analyse, das beste bislang entwickelte soziale Bewertungsverfahren. Da die Fakultätsaktivitäten in finanziellen Größen erfasst werden, liegt die Rechnung in Geldwerten und somit die Anwendung der NutzenKosten-Analyse nahe. Allerdings lassen sich die Schwächen verschiedener Bewertungsmethoden nicht vollständig überwinden, soweit das soziale Rechnungswesen auf der Verwendung monetärer Werte ${ }^{19}$ fußt. Viele der dort eingesetzten Bewertungsverfahren werden auch von den erreichbaren Informationen geprägt.

3. Falls das vorgeschlagene Buchführungssystem für mehrere Fakultäten oder die gesamte Universität eingesetzt werden soll, muss der Kontenrahmen angepasst werden. Auch die Ausgrenzungsregeln sind zu spezifizieren.

4. Erweiterungen des Kontenrahmens werden erforderlich, wenn gruppenspezifische Wohlfahrtsgewinne ermittelt werden sollen, etwa für Studentengruppen oder die Auftraggeber von Forschungsleistungen. Dann sind einige der Ausgrenzungsregeln gruppenspezifisch zu fassen. Zusätzliche Korrekturen der im kommerziellen Rechnungswesen ermittelten sozialen Nutzen und sozialen Kosten haben zu erfolgen und müssen im zusätzlichen sozialen Rechnungswesen Berücksichtigung erfahren. Wiederum lassen sich prinzipiell die gesamten Nettonutzen, die gesamten sozialen Vermögen und die gesamten sozialen Verpflichtungen ermitteln.

5. Einige Fachvertreter sind der Ansicht, dass ein soziales Rechnungswesen auf der Basis der NutzenKosten-Analyse zu viele Bewertungen erfordere und deshalb nicht geeignet sei, um die gesellschaftlichen Implikationen der Fakultätsaktivitäten aufzuzeigen. Die breite Zielstruktur der betrachteten Fakultät und ihre vielfältige Rolle in der Gesellschaft erfordern jedoch einen solchen weiten mit dem kaufmännischen Rechnungswesen kombinierten Ansatz.

6. Da das hier entwickelte gesellschaftsbezogene Rechnungswesen monetäre Bewertungen benutzt, wird der gesellschaftliche Erfolg mit der Wirtschaftslage und den Preisentwicklungen schwanken, z. B. bestand in Estland im Jahre 2006 eine Boomperiode, während im Jahre 2010 bei ähnlichem Aktivitätsumfang im Zuge der Wirtschaftskrise mit einem geringeren sozialen Erfolg zu rechnen ist, da die Einkommen und teilweise auch die Preise währen der Krise gesunken sind. ${ }^{20}$

19 Da die Buchungen im zusätzlichen sozialen Rechnungswesen nicht nach Einzelbelegen, sondern unter Verwendung von jährlichen Größen erfolgen, sind ca. 210 Größen mit inhaltlicher Wertbestimmung auf Zahlungsbereitschaftsansatzbasis zu buchen. Hier gelangen die Monetarisierungsverfahren zur Anwendung, für die ähnlich wie in den vergangenen zwei Jahrhunderte für das kaufmännische Rechnungswesen - viele Einzeldissertationen und Habilitationsschriften zu deren Eignung im Rahmen dieses Buchführungssystems verfasst werden können. Für Auskünfte über die Bewertung und Erfassung einzelner Buchungen stehen die Autoren gerne zur Verfügung.

20 Dies betraf auch die Einkommen der Mitarbeiter und des Lehr- und Forschungspersonals des öffentlichen Unternehmens Universität. 
Die zweite Gruppe von Argumenten betrifft die folgenden Schwächen:

1. Die Ansätze zum Bildungsrechnungswesen, die zurzeit Anwendung finden sind, wenig leistungsfähig. Dies trifft besonders für Fakultätsleistungen zu. Die meisten zugänglichen Bildungsevaluationen beschränken sich auf Berichte auf der Basis physischer Indikatoren sowie von Kosten mit geringer Beziehung zu den gesellschaftlichen oder administrativen Zielen einer Fakultät und den Beiträgen der einbezogenen Wirtschaftseinheiten zum sozialen Erfolg.

2. Die Anwendung und Entwicklung detaillierter Bewertungsverfahren ist aufwendig, aber sie erlauben Aussagen zu einer besseren Beurteilung der Aufgabenerfüllung der Fakultät. Die übliche Berichterstattung schildert Effekte auf Wirtschaftseinheiten, die mit der Fakultät verbunden sind sowie betroffene Interessentengruppen, ohne dass deren Beiträge zum gesellschaftlichen Erfolg ausgegrenzt werden. Ferner sind diese Berichte auch auf einzelwirtschaftliche Konkurrenz-, Bereichs- oder Managementziele ausgerichtet.

3. Falls mehrere Fakultäten, die Universität oder mehrere Universitäten, Fachhochschulen, Colleges dieses gesellschaftsbezogene Rechnungswesen einführen wollen, sind für gleiche Transaktionen gleiche Verbuchungs-, Bewertungs- und Ausgrenzungsverfahren anzuwenden, falls man die sozialen Erfolge der Institutionen vergleichen will. Dafür besteht zurzeit kein institutionell begründeter Zwang über Gesetzgebung, Verwaltungsverfahren und Buchführungsordnungen.

Somit resultieren vielseitige weiterführende Forschungsaufgaben im Bereich der Effekterfassung, z. B. genauere Abschreibungssätze für Wissensverluste der Bachelor-, Masterstudenten, der Doktoranden, Forscher und Professoren, und der Bewertungen im Bereich der Konsumentenrentenerfassungen, z. B. über bessere Schätzungen von Nachfragefunktionen für einzelne Leistungen, Verfahren zur Vermögensbewertung, Bewertungsverfahren zur Ermittlung der Zahlungsbereitschaft für einzelne Leistungsgruppen, der Zuordnung von Vorleistungen der Fakultät aufColleges oder der Universität auf Fakultäten. Weiterhin sollte in der hiesigen Fakultät über das Jahr 2006 hinaus die Buchführung für die Jahre 2007, 2008, 2009, 2010 und 2011 fortgeführt werden, um die Grundlage und Ideen zur Verbesserung des Buchführungssystems zu erzielen. ${ }^{21}$ Dann besteht eine bessere Grundlage zur Beurteilung der Leistungsfähigkeit des Ansatzes und in welcher Weise die ,stakeholder“ Fakultät, Universität, Ministerium, Studenten, Beschäftigte, Politiker von dieser Variante des gesellschaftsbezogenen Rechnungswesens Managementvorteile erlangen. Auch die Implementierungsbarrieren, z. B. aufgrund mangelnden Verständnisses oder gruppenspezifisch befürchteter Nachteile bei der Zumessung von Einkommen, lassen sich dann besser abschätzen.

\section{Literaturverzeichnis}

Abt, Clark C. (1972), Social Audits: The State of the Art, New York.

Ahlheim, Michel und Manfred Rose (1989), Messung individueller Wohlfahrt, Heidelberg.

Ammermüller, Andreas und Dieter Dohem (2004), Individuelle und soziale Erträge von Bildungsinvestitionen, Studien zum deutschen Innovationssystem, Nr. 1-2004, Berlin, Köln.

Azuma, Kentaro (2007), Ökobilanzierung und Periodisierung, Berlin.

Bauder, Artur und André Jungen (2009), Ansatz- und Bewertungsprobleme bei der Aufstellung der Eröffnungsbilanz staatlicher Hochschulen am Beispiel von Nordrhein-Westfalen, in: Zeitschrift für öffentliche und gemeinwirtschaftliche Unternehmen, 32. Jg., Heft 2, S. 113-132.

Belfield, Cive R. (2000), Economic Principles for Education, Cheltenham, Northampton.

21 Ein Forschungsprojekt des estnischen Wissenschaftsministeriums zum Universitätsmanagement, eine Dissertation und zukünftige Masterstudien dienen der Weiterentwicklung des vorgestellten Ansatzes. 
Birch, Stephen und Amiram Gafni (1992), Cost Effectiveness/Utility Analysis - Do Current Decision Rules Lead Us to where We Want to Be? Journal of Health Economics, Vol. 11, S. 279-296.

Bräuning, Dietmar (2004), Rechnungswesen und Steuerung öffentlicher Verwaltungen, in: Betriebswirtschaftliche Forschung und Praxis, 56. Jg., Heft 4, S. 309-322.

Bräuning, Dietmar und Peter Eichhorn (2002), Evaluation and Accounting Standards in Public Management, BadenBaden.

Brent, Robert.J. (2004), Cost-Benefit Analysis and Health Care Evaluations, Cheltenham.

Breyer, Friedrich und Reiner Leidl (1997), Wozu dient Evaluation im Gesundheitswesen? in: Managed Care, hrsg. von Eckhard Knappe, Baden-Baden, S. 121-138.

Brüngger, Heinrich (1974), Die Nutzen-Kosten-Analyse as Instrument der Planung im Gesundheitswesen, Zürich.

Brummert, Lee R. (1969), An Overview, in: Human Resource Accounting, Development and Implementation in Industry, Foundation for Research in Human Behavior, hrsg. von Lee R. Brummert, Eric G. Flarnholtz und William C. Pyle, Ann Arbor, S. 11-21.

Bullinger, Monika (1998), Der SF-36 Health Survey als krankheitsübergreifendes Profilinstrument, in: Gesundheitsökonomische Evaluationen, hrsg. von Oliver Schöffski, Petra Glaser und J. Mathias Graf v. d. Schulenburg, Berlin, et al., S. 177-187.

Claes, Christa (1998), Möglichkeiten und Grenzen der Datenerfassung, in: Gesundheitsökonomische Evaluationen, hrsg. von Oliver Schöffski, Petra Glaser und J. Mathias Graf v. d. Schulenburg et al., Berlin S. 99-114.

Claes, Christa, Uber, Andrea und Wolfgang Greiner, (1998), Der EuroQol als krankheitsspezifisches Indexinstrument, in: Gesundheitsökonomische Evaluationen, hrsg. von Oliver Schöffski, Petra Glaser und J. Mathias Graf v. d. Schulenburg et al, Berlin, S. 193-201.

Cohn, Elchanan und Terry Geske (1992), Private Nonmonetary Returns to Investment in Higher Education, in: The Economics of American Higher Education, hrsg. von William E. Becker und R. Darell Lewis, S. 173-196.

Conrads, Michael (1976) Human Resource Accounting, Wiesbaden.

Department of Bookkeeping (2006), Data of 2006, University of Tartu, Tartu.

Dierkes, Meinolf (1974), Die Sozialbilanz, Frankfurt.

Eichhorn, Peter (1974), Gesellschaftsbezogene Unternehmensrechnung, Göttingen.

Elliot-Jones, M. F. (1973), Matrix Methods in Corporate Accounting, in: Corporate Social Accounting, hrsg. von Meinold Dierkes und Roger A Bauer, New York, Washington, London, S. 351-36.

Faculty of Economics and Business Administration (2007), Self-Analysis Report, University of Tartu, Tartu.

Fischer-Winkelmann, Wolf F. (1980), Gesellschaftsorientierte Unternehmensrechnung, München.

Flamholtz, Eric G. (1971), A Model for Human Resource Valuation: A Stochastic Process with Service Rewards, in: The Accounting Review, Vol. 46, S. 253-267.

Flores, Nicholas E. (2003), Conceptional Framework for Nonmarket Valuation, in: A Primer on Nonmarket Valuation, hrsg. von Particia A. Champ, Kevin.J. Boyle und Thomas. C. Browns, Dordrecht, S. 27-58.

Friedrich, Peter (1969), Volkswirtschaftliche Investitionskriterien für Gemeindeunternehmen, Tübingen 1969.

Friedrich, Peter (1991), Problematik des Kontenrahmens eines gesellschaftsbezogenen Rechnungswesens, in: Dienstprinzip und Erwerbsprinzip, hrsg. von Peter Faller und Dieter Witt, Baden-Baden, S. 139-235.

Friedrich, Peter und Sakari Jutila (2001), Aspects of Policies of Regional Competition, in: Policies of Regional Competition, hrsg. von Peter Friedrich und Sakari Jutila, Sakari, Baden-Baden, S. 13-60.

Friedrich, Peter, Jahn, Klaus., Valjak, Günther und, Heinrich G. Wonnemann (1993), Ein gesellschaftsbezogenes Rechnungswesen am Beispiel der Stadthalle Osnabrück, in: Zeitschrift für öffentliche und gemeinnützige Unternehmen, Vol. 16, S. 245-247.

Friedrich, Peter, Feng, Xiao, Jahn, Klaus, Valjak, Günther und Heinrich G. Wonnemann (2000), Economic Effects of Locating Tourist Facilities, in: Tourism Sustainability and Territorial Organisation, hrsg. von APDR, Coimbra, S. 143-174.

Friedrich, Peter, Kosiński, Janusz A, und Ingrid Türk (2003), A Standard Chart of Social Accounts for Ecological Banks, in: Finance and Natural Environment, hrsg. von Leszek Dziawgo und Danuta Dziawgo, Torun, S. 281-322.

Genduso, Laura A. und James G. Kotsanos (1996), Review of Health Economic Guidelines in the Form of Regulations, Principles, Policies, and Positions, in: Drug Information Journal, Vol. 30, S. 1003-1016.

Graaff, Jan.de V. (1963), Theoretical Welfare Economics, Cambridge.

Graf von der Schulenburg, J. Mathias (2007), Innovation im Deutschen Gesundheitssystem: die Kosten-NutzenAnalyse, in: Öffentliche Finanzen und Gesundheitsökonomie, hrsg. von Hans Adam, Cornelia Behrens, Dirk Göpffrath und Beate Jochimsen, Baden-Baden, S. 309-322.

Graf von Stillfried, Dominik und Gerd Glaeske (1998), Ökonomische Evaluation der medizinischen Versorgung Standortbestimmung aus Sicht der Barmer Ersatzkasse, in: Gesundheitsökonomische Evaluationen, hrsg. von Oliver Schöffski, Petra Glaser und J. Mathias Graf v. d. Schulenburg, et al., Berlin, S. 285-306.

Gray, Rob, Bebbington, Jan und Sue Gray (Hrsg.) (2010), Social and Environmental Accounting, Vol. I-IV, Los Angeles, Sondon, New Delhi, Singapore, Washington D.C.

Greiner, Wolfgang (1998), Die Berechnung von Kosten und Nutzen im Gesundheitswesen, in: Gesundheitsökonomische Evaluationen, hrsg. von Oliver Schöffski, Petra Glaser und J. Mathias Graf von der Schulenburg, Berlin, et al., S. 55-68. 


\section{Peter Friedrich und Diana Eerma}

Greiner, Wolfgang und Oliver Schöffski (1998), Grundprinzipien einer Wirtschaftlichkeitsrechnung, in: Gesundheitsökonomische Evaluationen, hrsg. von Oliver Schöffski, Petra Glaser und J. Mathias Graf v. d. Schulenburg, et al., Berlin, S. 79-98.

Greiner, Wolfgang, Uber, Andrea und J. Mathias Graf von der Schulenburg. (1996), Ökonomische Bewertung von Leistungen im Gesundheitswesen, in: Public Health - Gesundheit im Mittelpunkt, hrsg. von, Ulla Walter und Paris Walter, Meran, S. 70-77.

Hanusch, Horst (1987), Nutzen-Kosten-Analyse, München.

Haveman, Robert H. und Barbara L. Wolfe, (1984), Schooling and Economic Well-Being: The Role of Nonmarket Effects, in: The Journal of Human Resources, Vol. 19, S. 377-407.

Heiling, Jens (2007), Rechnungslegung staatlicher Hochschulen, Berlin.

Helmig, Bernd (2005), Ökonomischer Erfolg in öffentlichen Krankenhäusern, Berlin.

Henke, Klaus Kirk (1986), Die direkten und indirekten Kosten von Krankheiten in der Bundesrepublik Deutschland im Jahre 1980, in: Finanzierung im Gesundheitswesen, hrsg. von Klaus Dirk Henke und Ingo Metze, Stuttgart, S. 213-262.

Hermanson, Roger H. (1964), Accounting for Human Assets, Michigan State University, Bureau of Business and Economic Research, Occasional Paper No. 14, East Lansing.

Holfelder, Georg (1998), Standortbestimmung zu gesundheitsökonomischen Evaluationen aus der Sicht Fachärztlicher Berufsverbände, in: Gesundheitsökonomische Evaluationen, hrsg. von Oliver Schöffski, Petra Glaser und J. Mathias Graf von der Schulenburg et al., Berlin, S. 371-384.

Hunter, David J. und Gelian Fairfield (1997), Disease Management, in: British Medical Journal, Vol. 315, S. 50-53.

Inter-Agency Committee on Waterresources (1958), Proposed Practices for Economic Analysis of River Basin Projects prepared by the Subcommittee, Washington.

Kaya, Ugur und Yayla, Hilmi Erdogan (2007), Remembering Thirty-five Years of Social Accounting: A Review of the Literature and the Practice, MPRA, München.

Kämmerer, Wolfgang (1998), Standortbestimmung und Ausblick aus Sicht der Krankenhausapotheken, in: Gesundheitsökonomische Evaluationen, in: Finanzierung im Gesundheitswesen, hrsg. von Klaus Dirk Henke und Ingo Metze, Stuttgart Schöffski, et al., Berlin, S. 401-414.

Konsensgruppe,,Gesundheitsökonomie"(1996), Empfehlungen zur gesundheitsökonomischen Evaluation - Hannoveraner Konsens -, in: Zeitschrift für Allgemeinmedizin, Vol. 72, S. 485-490.

Kossow, K.-D. (1998), Gesundheitsökonomische Evaluationen aus hausärztlicher Sicht,. in: Gesundheitsökonomische Evaluationen, hrsg. von Oliver Schöffski, Petra Glaser und J. Mathias Graf v. d. Schulenburg, et al., Berlin, S. 351-370.

Küpper, Hans Ulrich (2001), Rechnungslegung von Hochschulen, in: Betriebswirtschaftliche Forschung und Praxis, 53 Jg., Heft 6, S. 578-592.

Leslie, Larry und R. Darell Lewis (2003), Economic Magnet and Multiplier Effects of the University of Minnesota, in: The Public Research University, hrsg. von R. Darnell Lewis und James C. Heran, Lanham, New York, Oxford, S. 125-149.

Linowes, David F. (1961), Socio Economic Accounting, in: Journal of Accountancy, Vol. 14, S. 37-42.

Meyke, Udo (1973), Cost-Effectiveness-Analysis als Planungsinstrument, Göttingen 1973.

Mintrop, Angelika (1976), Gesellschaftsbezogene Rechenschaftslegung, Zürich.

Monsen, R. Joseph (1972), Social Responsibility and the Corporation, Alternatives for the Future of Capitalism, in: Journal of Economic Issues, Vol. 6, S. 125-141.

Mühlenkamp, Holger (2007), Vom neuen öffentlichen Rechnungswesen zu einer gesellschaftsbezogenen Rechnungslegung, in: Stand und Perspektiven der Öffentlichen Betriebswirtschaftslehre II, hrsg. von Dietmar. Bräuning und Dorothea Greiling, Berlin, S. 705-718.

Müller-Bohn, Thomas, Ulrich, Volker (2000), Pharmaökonomie, Stuttgart.

Neubauer, F. Friedrich (1974), Neuere Entwicklungen im amerikanischen Rechnungswesen: Das Human Resource Accounting, in: Die Unternehmung, Vol. 28, S. 261-280.

Partha, Dasguptha, Sen, Amartya und Stephen Marglin (1972), Guidelines for Project Evaluation, New York.

Psacharopoulos, George (1987), The Cost-Benefit Model, in: Economics of Education - Research and Studies, hrsg. von George Psacharopoulos, Oxford et al., S. 342-347.

Psacharopoulos, George (1987 a), Economics of Education, Oxford et al.

Rychlik, Reinhard. (1999), Gesundheitsökonomie, Stuttgart.

Sachverständigenrat für die konzertierte Aktion im Gesundheitswesen (1998), Gesundheitswesen in Deutschland, Sondergutachten, Kostenfaktor und Zukunftsbranche, Band I, Baden-Baden.

Schauer, Reinbert (2007), Renaissance der Sozialbilanzen, in: Stand und Perspektiven der Öffentlichen Betriebswirtschaftslehre II, Dietmar Bräuning und Dorothea Greiling, Berlin, S. 719-728.

Schmitz, Bernd (1980), Gesellschaftsbezogene Rechnungslegung für Altenpflegeheime, Baden-Baden.

Schöffski, Oliver (1990), Wirtschaftlichkeitsuntersuchungen von Arzneimitteln, Prinzipien, Methoden und Grenzen der Gesundheitsökonomie, Hannover.

Schöffski, Oliver (1998) Nutzentheoretische Lebensqualitätsmessung, in: Gesundheitsökonomische Evaluationen, hrsg. von Oliver Schöffski, Petra Glaser und J. Mathias Graf v. d. Schulenburg,), Berlin et al. S. 129-159. 


\section{Ein Kontenrahmen für eine "gesellschaftsbezogene Rechnungslegung"}

Schöffski, Oliver, und J Mathias Graf von der Schulenburg (Hrsg.) (2002), Gesundheitsökonomische Evaluationen, Berlin et al.

Schöffski, Oliver und Andrea Uber (1998), Grundformen gesundheitsökonomischer Evaluation, in: Gesundheitsökonomische Evaluationen, hrsg. Von Oliver Schöffski, Petra Glaser und J. Mathias Graf v. d. Schulenburg, Berlin, et al, S. 69-78.

Schöffski, Oliver, Glaser, Petra und J. Mathias Graf v. d. Schulenburg (Hrsg.) (1998), Gesundheitsökonomische Evaluationen, Berlin et al.

Schwalbach, Joachim und Anja Schwerk (2008), Corporate Governance und Corporate Citizenship, in: Handbuch Corporate Citizenship, hrsg. von Andre Habich, Rene Schmidpeter und Martin Neureiter, Berlin, S. 71-85.

Schwalbach, Joachim, Schwerk, Anja und Daniel Smuda (2009), Stadtrendite - Der gesellschaftliche Nutzen von Wohnungsunternehmen, in: Neue Grenzen städtischer Wirtschaftstätigkeit: Ausweitung versus Abbau?, hrsg. von Peter Haug und Martin Rosenfeld, Baden-Baden, S. 137-148.

Seidel, Eberhard und Stefan Zenus (1990), Ökologisches Rechnungswesen, Literaturstudie für den VDI e.V., Manuskript, Düsseldorf.

Spraul, Katharina (2006), Bildungsrendite als Zielgröße für das Hochschulmanagement, Berlin.

Statistisches Bundesamt (1998), Gesundheitsbericht für Deutschland, Stuttgart

Thurow, Lester C. (1999), Building Wealth, New York.

Tengs, Tammy O., Adams, Miriam E und Joseph E. Pliskin et al. (1995), Five-Hundred Life-Saving Interventions and their Cost-Effectiveness, in: Risk Analysis, Vol. 15, S. 369-390.

Tsimopoulos, Dimitrios. (1989), Gesellschaftsbezogenes Rechnungswesen für Energieversorgungsunternehmen, Frankfurt.

University of Tartu (2006), Annual Report 2006, University of Tartu, Tartu.

Waltenberger, Monika (2006), Rechnungslegung staatlicher Hochschulen: Prinzipien, Struktur und Gestaltungsprobleme, München.

Weinstein, Milton C. (1990), Principles of Cost-Effective Resource Allocation in Health Care Organisations, in: International Journal of Technology Assessment in Health Care, Vol. 6, S. 93-103.

Weisbrod, Burton A (1964), External Benefits of Public Education, Princeton 1964.

Westlund, Hans (2006), Social Capital in the Knowledge Economy, Berlin, Heidelberg, New York.

Wille, Eberhard (1996), Anliegen und Charakteristika einer Kosten-Nutzen-Analyse, in: Ökonomie in der Medizin, hrsg. von J. Mathias Graf von der Schulenburg, Stuttgart, New York, S. 1-16.

Wonnemann, Heinrich G. (1989), Budgeteffekte der Standortwahl von Verwaltungen, Baden-Baden.

Wysocki, Klaus (1981), Sozialbilanzen, Stuttgart, New York.

Zangemeister, Christof (1976): Nutzwertanalyse in der Systemtechnik - Eine Methodik zur multidimensionalen Bewertung und Auswahl von Projektalternativen München. 